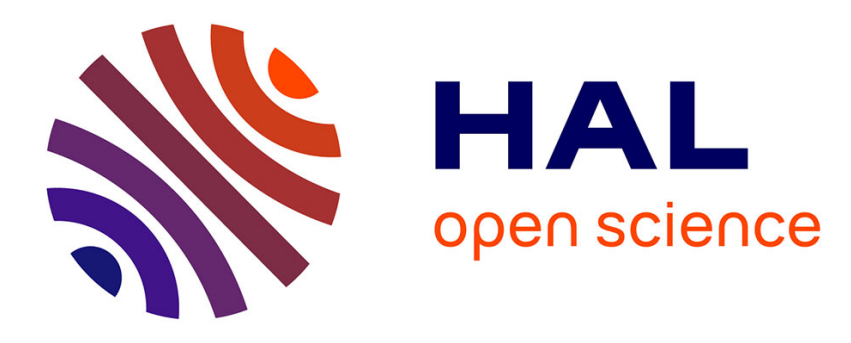

\title{
Boundary element based formulations for crack shape sensitivity analysis \\ Marc Bonnet
}

\section{To cite this version:}

Marc Bonnet. Boundary element based formulations for crack shape sensitivity analysis. Engineering Analysis with Boundary Elements, 2001, 25, pp.347-362. 10.1016/S0955-7997(01)00025-X . hal00092385

\section{HAL Id: hal-00092385 \\ https://hal.science/hal-00092385}

Submitted on 9 Aug 2008

HAL is a multi-disciplinary open access archive for the deposit and dissemination of scientific research documents, whether they are published or not. The documents may come from teaching and research institutions in France or abroad, or from public or private research centers.
L'archive ouverte pluridisciplinaire HAL, est destinée au dépôt et à la diffusion de documents scientifiques de niveau recherche, publiés ou non, émanant des établissements d'enseignement et de recherche français ou étrangers, des laboratoires publics ou privés. 


\title{
Boundary element based formulations for crack shape sensitivity analysis*
}

\author{
Marc BonneT \\ Laboratoire de Mécanique des Solides (UMR CNRS 7649) \\ Ecole Polytechnique, F-91128 Palaiseau Cedex, France. \\ bonnet@lms.polytechnique.fr
}

\begin{abstract}
The present paper addresses several BIE-based or BIE-oriented formulations for sensitivity analysis of integral functionals with respect to the geometrical shape of a crack. Functionals defined in terms of integrals over the external boundary of a cracked body and involving the solution of a frequency-domain boundary-value elastodynamic problem are considered, but the ideas presented in this paper are applicable, with the appropriate modifications, to other kinds of linear field equations as well. Both direct differentiation and adjoint problem techniques are addressed, with recourse to either collocation or symmetric Galerkin BIE formulations. After a review of some basic concepts about shape sensitivity and material differentiation, the derivative integral equations for the elastodynamic crack problem are discussed in connection with both collocation and symmetric Galerkin BIE formulations. Building upon these results, the direct differentiation and the adjoint solution approaches are then developed. In particular, the adjoint solution approach is presented in three different forms compatible with BEM analysis of crack problems, based on the discretized collocation BEM equations, the symmetric Galerkin BEM equations and the direct and adjoint stress intensity factors, respectively. The paper closes with a few comments.
\end{abstract}

*Engineering Analysis with Boundary Elements, 25, 347-362 (2001) 


\section{Introduction}

The consideration of sensitivity analysis of integral functionals with respect to shape parameters arises in many situations where (part of) a geometrical domain is either unknown or variable. Shape optimization and inverse problems are the most obvious instances, but not the only ones; for instance, the energy release rate, a basic concept of fracture mechanics, is mathematically defined as (minus) the derivative of the potential energy at equilibrium with respect to crack front perturbations. For these reasons, the numerical evaluation of sensitivities of functionals with respect to shape perturbations is clearly an important issue. The present paper is specifically concerned with boundary element-based methods for computing the sensitivity of integral functionals with respect to crack shape perturbations.

This goal is achievable by resorting to either finite-difference methods, considering small but finite domain perturbations, or analytical differentiation followed by discretization. The analytical approach is a priori clearly superior in terms of both accuracy and efficiency. It relies on either the adjoint variable approach or a direct differentiation of the field equations formulated in weak or BIE fashion. A substantial research effort has been devoted in the last decade or so to various formulations and applications of sensitivity analyses based on analytical differentiation with respect to shape parameters, or on the related mathematical concept of domain derivative [41,42]. As a result, these concepts are successfully applied to more and more engineering problems (see e.g. $[21,29,30]$, among a quite abundant literature).

Further, since (the shape of) the boundary plays a key role in problems with variable or unknown domains, it is often found convenient, or even essential, to resort to the boundary element method (BEM). Both the adjoint problem $[1,4,17-20,34]$ and the direct differentiation approach $[2,5,23,32$, $33,36,37,39,45]$ have been investigated in connection with BEMs (see also the journal special issue [15]). Besides, defect identification problems are sometimes solved using successive linearizations of measurement residuals $[24,25]$.

The present paper addresses several BIE-based or BIE-oriented formulations for sensitivity analysis of integral functionals with respect to the geometrical shape of a crack. The functionals considered here are defined in terms of integrals over the external boundary involving the solution of a linear boundary-value problem in frequency-domain elastodynamics. Both direct differentiation and adjoint problem techniques are addressed, with recourse to either collocation or symmetric Galerkin BIE formulations. Following the statement of a generic direct elastodynamic problem (section 2) and a review of some basic concepts about shape sensitivity and material differentiation (section 3), integral identities in derivative form are established in (section 4). These results allow to 
formulate the derivative integral equations for the elastodynamic crack problem defined in section 2 in connection with collocation BIE (section 5) and symmetric Galerkin BIE (section 6). Building upon these results, the direct differentiation and the adjoint solution approaches are discussed in sections 7 and 8 respectively. In particular, the adjoint solution approach is presented in three different forms compatible with BEM analysis of crack problems. The paper closes with a few comments (section 9).

\section{The direct problem}

Let us consider, in the three-dimensional Euclidean space $\mathbb{R}^{3}$ equipped with a Cartesian orthonormal basis $\left(\boldsymbol{e}_{1}, \boldsymbol{e}_{2}, \boldsymbol{e}_{3}\right)$, an elastic body $\Omega \in \mathbb{R}^{3}$ of finite extension, externally bounded by the closed surface $S$ and containing a crack $\Gamma$. The unit normal $\boldsymbol{n}$ to $\Gamma$ is oriented along the $\Gamma^{-} \rightarrow \Gamma^{+}$ direction, where $\Gamma^{+}, \Gamma^{-}$are the two crack faces (the outward normal to $\Gamma^{ \pm}$is thus $\mp \boldsymbol{n}$ ). The displacement $\boldsymbol{u}$, strain $\boldsymbol{\varepsilon}$ and stress $\boldsymbol{\sigma}$ in $\Omega$ are related by the field equations:

$$
\begin{gathered}
\operatorname{div} \boldsymbol{\sigma}+\rho \omega^{2} \boldsymbol{u}=\mathbf{0} \\
\boldsymbol{\sigma}=\boldsymbol{C}: \boldsymbol{\varepsilon} \\
\boldsymbol{\varepsilon}=\frac{1}{2}\left(\boldsymbol{\nabla} \boldsymbol{u}+\nabla^{T} \boldsymbol{u}\right)
\end{gathered}
$$

where $\boldsymbol{C}$ denotes the fourth-order elasticity tensor, given in the isotropic case by:

$$
C_{a b c d}=\mu\left(\frac{2 \nu}{1-2 \nu} \delta_{a b} \delta_{c d}+\delta_{b d} \delta_{a c}+\delta_{b c} \delta_{a d}\right)
$$

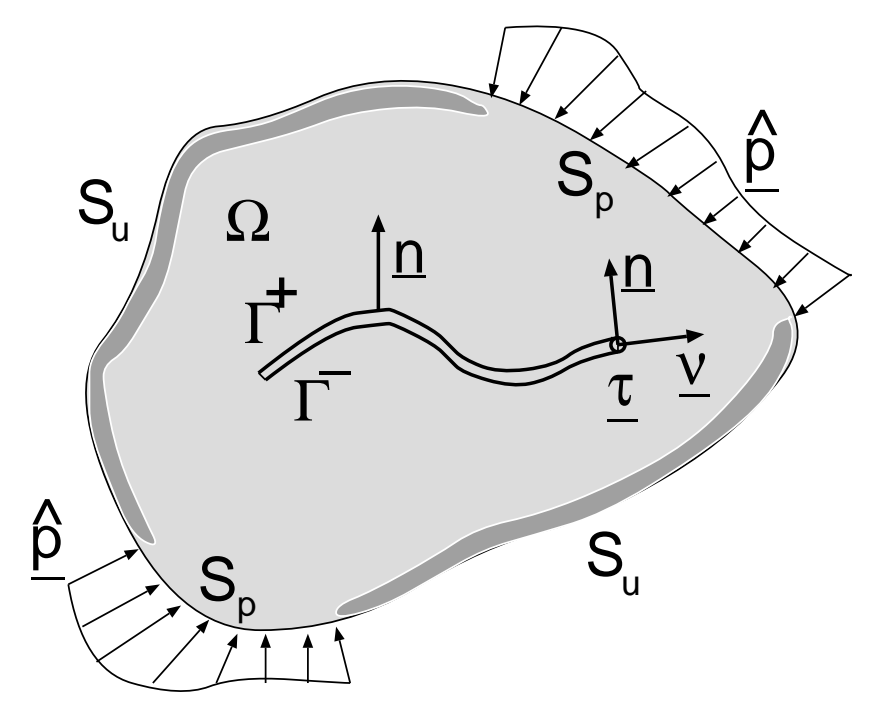

Figure 1: Cracked elastic solid $\Omega$ and boundary conditions. 
where $\mu$ and $\nu$ denote respectively the shear modulus and the Poisson ratio. Besides, displacements and tractions are prescribed on the portions $S_{u}$ and $S_{p}=S \backslash S_{u}$ of $S$, while the crack surface $\Gamma$ is stress-free:

$$
\begin{aligned}
& \boldsymbol{u}=\hat{\boldsymbol{u}} \quad\left(\text { on } S_{u}\right) \\
& \boldsymbol{p}=\hat{\boldsymbol{p}} \quad\left(\text { on } S_{p}\right) \\
& \boldsymbol{p}=\mathbf{0} \quad(\text { on } \Gamma)
\end{aligned}
$$

where $\boldsymbol{p} \equiv \boldsymbol{\sigma} . \boldsymbol{n}$ is the traction vector, defined in terms of the outward unit normal $\boldsymbol{n}$ to $\Omega$. For a given location of the crack, the field equations (1) and boundary conditions (2) define the direct problem.

\section{Material derivative in a shape perturbation}

To investigate the effect of crack shape perturbations, the shape of the body $\Omega$ is assumed to depend on a parameter $t$ (a fictitious, non-physical 'time') through a continuum kinematics-type Lagrangian description. The unperturbed, 'initial' configuration $\Omega$ is conventionally associated with $t=0$ :

$$
\boldsymbol{x} \in \Omega \rightarrow \boldsymbol{x}^{t}=\boldsymbol{\Phi}(\boldsymbol{x}, t) \in \Omega(t) \quad \boldsymbol{\Phi}(\boldsymbol{x}, 0)=\boldsymbol{x}
$$

All 'time' derivatives will be implicitely taken at $t=0$, i.e. the first-order effect of infinitesimal perturbations of $\Omega \equiv \Omega(0)$ is considered. The geometrical transformation $\mathbf{\Phi}(\cdot ; t)$ must possess a strictly positive Jacobian for $t \geq 0$. A given domain evolution considered as a whole admits infinitely many different representations (3).

\subsection{Material derivative of scalar or tensor fields}

Differentiation of field variables and integrals in a domain perturbation is a well-documented subject, see e.g. Petryk and Mroz [40], Sokolowski and Zolesio [42]; a few basic concepts and results are recalled now. The initial transformation velocity $\boldsymbol{\theta}$ is defined by:

$$
\boldsymbol{\theta}(\boldsymbol{x})=\left.\frac{\partial \boldsymbol{\Phi}}{\partial t}\right|_{t=0}
$$

The 'material' (or 'Lagrangian') derivative at $t=0$ of a field quantity $f(\boldsymbol{x}, t)$ in a geometrical transformation, denoted by $\stackrel{\star}{f}$, is defined by:

$$
\stackrel{\star}{f}=\lim _{t \searrow 0} \frac{1}{t}\left[f\left(\boldsymbol{x}^{t}, t\right)-f(\boldsymbol{x}, 0)\right]=\frac{\partial f}{\partial t}+\nabla f . \boldsymbol{\theta}
$$


The material derivative of the gradient of a field quantity is given by:

$$
(\nabla f)^{\star}=\nabla \stackrel{\star}{f}-\nabla f \cdot \nabla \boldsymbol{\theta}
$$

\subsection{Material derivative of surface integrals}

The material derivatives of the unit normal $\boldsymbol{n}$ and the surface differential element $\mathrm{d} S$ on a material surface $S_{t}=\boldsymbol{\Phi}(S ; t)$ are given (see e.g. [40]) by:

$$
\begin{gathered}
\stackrel{\star}{\mathrm{d}} S=\operatorname{div}_{S} \boldsymbol{\theta} \mathrm{d} S=D_{r} \theta_{r} \mathrm{~d} S \\
\stackrel{\star}{\boldsymbol{n}}=-\boldsymbol{n} \cdot \nabla_{S} \boldsymbol{\theta}=-n_{b} D_{a} \theta_{b} \boldsymbol{e}_{a}
\end{gathered}
$$

in terms of the surface gradient $\nabla_{S}$ and the surface divergence $\operatorname{div}_{S}$ :

$$
\begin{gathered}
\nabla_{S} f=\nabla f-(\nabla f . \boldsymbol{n}) \boldsymbol{n}=\left(f_{, i}-n_{i} f_{, n}\right) \boldsymbol{e}_{i} \equiv\left(D_{i} f\right) \boldsymbol{e}_{i} \\
\operatorname{div}_{S} \boldsymbol{u}=\operatorname{div} \boldsymbol{u}-(\boldsymbol{\nabla u} . \boldsymbol{n}) . \boldsymbol{n}=D_{i} u_{i}
\end{gathered}
$$

Combining equations (7) and (8) then yields another useful property:

$$
\left(n_{a} \mathrm{~d} S\right)^{\star}=\left(n_{a} D_{b}-n_{b} D_{a}\right) \theta_{b} \mathrm{~d} S=e_{a b c} R_{c} \theta_{b} \mathrm{~d} S
$$

where $e_{a b c}$ is the permutation tensor and $R_{i} f$ denotes the $i$-th component of the surface curl of a scalar function $f$ :

$$
R_{i} f=e_{i j k} n_{j} f_{, k}
$$

which is a tangential differential operator [35] associated to a variant of the Stokes formula, valid for any piecewise regular surface $S$ and continuous, piecewise differentiable $f$ :

$$
\int_{S} R_{i} f \mathrm{~d} S=\int_{\partial S} \tau_{i} f \mathrm{~d} S \quad(=0 \text { if } S \text { is closed })
$$

(the unit tangent $\boldsymbol{\tau}$ to $\mathrm{d} S$ is such that $\boldsymbol{\nu}=\boldsymbol{n} \times \boldsymbol{\tau}$ points towards the exterior of $S$ ).

Then, for a generic surface integral $I(p)$ :

$$
I(f, S ; t)=\int_{S(t)} f(\boldsymbol{x}, t) \mathrm{d} S
$$

one has, using (7):

$$
\frac{d I}{d t}=\stackrel{\star}{I}=\int_{S}\left\{\stackrel{\star}{f} \mathrm{~d} S+f(\mathrm{~d} S)^{\star}\right\}=\int_{S}\left\{\stackrel{\star}{f}+f \operatorname{div}_{S} \boldsymbol{\theta}\right\} \mathrm{d} S
$$

Using Eq. (11) yields an useful variant of the latter identity:

$$
\frac{d}{d t} \int_{S} f n_{a} \mathrm{~d} S=\int_{S}\left\{\stackrel{\star}{f} n_{a}+f e_{a b i} R_{i} \theta_{b}\right\} \mathrm{d} S
$$

Note that $\stackrel{\star}{f}$ and $\stackrel{\star}{I}$ depend linearly on $\boldsymbol{\theta}$ if $\frac{\partial f}{\partial t}=0$. 


\section{Strongly singular and hypersingular derivative integral identi- ties}

The material differentiation formulas are, at first glance, applicable only to nonsingular or at most weakly singular integrals on moving surfaces. Since BIE formulations are either strongly singular or hypersingular, one possibility is thus to first rewrite the integral equations using indirect regularization, a technique which is now well-documented (see e.g. [10,31] and the survey paper [43]) and then apply material differentiation to the regularized BIE in a straightforward manner. This approach is developed for displacement-type integral equations, up to second-order material derivatives, in [5] for three-dimensional problems and in [45] for two-dimensional problems; see also [32] and [39]. Moreover, the material differentiation of surface integrals has been shown in an earlier work [7] to be valid even for the strongly- and hypersingular integral operators usually encountered in non-regularized BIE formulations; this allows to work within the framework of so-called direct approaches [26] and validates a posteriori the approach followed in e.g. [2, 23,33].

To discuss the material differentiation of BIEs, one can thus start from BIE formulations in either regularized or direct forms. In this paper, the latter choice is adopted, for two main reasons. First, this will allow us to emphasize again the correctness of taking material derivatives of the differentiability (in domain perturbations) of strongly singular or hypersingular integrals. Second, since the regularization of hypersingular BIEs introduces many additional terms in the formulations, the material differentiation leads to very lengthy equations, whereas the non-regularized viewpoint yields somewhat more compact formulas at the material derivative level.

In sections 4.1 and 4.2 below, basic derivative integral identities arising from strongly singular and hypersingular BIE formulations are stated for an uncracked domain $\Omega$. The results established therein will then be applied to formulate derivative BIEs for elastic solids with cracks, first in collocation form (section 5), then in symmetric Galerkin form (section 6).

\subsection{Material derivative of strongly singular BIE}

Following the approach and notations of Guiggiani [26], let $\tilde{\boldsymbol{x}}$ be a fixed point on the boundary $\partial \Omega$ of a three-dimensional domain $\Omega$. We consider an exclusion neighbourhood $v_{\varepsilon}(\tilde{\boldsymbol{x}})$ of $\tilde{\boldsymbol{x}}$, of radius $\leq \varepsilon$ (Figure 2). For any $\varepsilon>0, \tilde{\boldsymbol{x}}$ is always an external point for the domain $\Omega_{\varepsilon}(\tilde{\boldsymbol{x}})=\Omega-v_{\varepsilon}(\tilde{\boldsymbol{x}})$ whose boundary $\partial \Omega-e_{\varepsilon}$ is given by

$$
\partial \Omega-e_{\varepsilon}=\left(\partial \Omega-e_{\varepsilon}\right)+s_{\varepsilon}=S_{\varepsilon}+s_{\varepsilon}
$$

where $s_{\varepsilon}=\Omega \cap \partial v_{\varepsilon}, e_{\varepsilon}=\partial \Omega \cap \bar{v}_{\varepsilon}$, and $S_{\varepsilon}=\partial \Omega-e_{\varepsilon}$. 
The usual strongly singular BIE formulation is the limiting form when $\varepsilon \rightarrow 0$ of the identity:

$$
\int_{S_{\varepsilon}+s_{\varepsilon}}\left\{T_{a}^{k}(\tilde{\boldsymbol{x}}, \boldsymbol{x}) u_{a}(\boldsymbol{x})-U_{a}^{k}(\tilde{\boldsymbol{x}}, \boldsymbol{x}) p_{a}(\boldsymbol{x})\right\} d S_{x}=0
$$

where $U_{a}^{k}(\tilde{\boldsymbol{x}}, \boldsymbol{x})$ and $\Sigma_{a b}^{k}(\tilde{\boldsymbol{x}}, \boldsymbol{x})$ are the components of the elastodynamic fundamental solution (Appendix A.1) and $T_{a}^{k}(\tilde{\boldsymbol{x}}, \boldsymbol{x})=\Sigma_{a b}^{k}(\tilde{\boldsymbol{x}}, \boldsymbol{x}) n_{b}(\boldsymbol{x})$ is the fundamental traction vector.

Under the sufficient regularity assumption $u \in C^{0, \alpha}$ at the singular point $\tilde{\boldsymbol{x}}$, one has

$$
\lim _{\varepsilon \rightarrow 0} \int_{s_{\varepsilon}}\left[u_{a}(\boldsymbol{x})-u_{a}(\tilde{\boldsymbol{x}})\right] T_{a}^{k}(\tilde{\boldsymbol{x}}, \boldsymbol{x}) d S_{x}=0
$$

Carrying out the limiting process in the statements (16) using a spherical exclusion neighbourhood $v_{\varepsilon}$ then leads to the well-known strongly singular BIE formulation (see Guiggiani and Gigante [27]):

$$
c_{k a}(\tilde{\boldsymbol{x}}) u_{a}(\tilde{\boldsymbol{x}})+\int_{S} T_{a}^{k}(\tilde{\boldsymbol{x}}, \boldsymbol{x}) u_{a}(\boldsymbol{x}) d S_{x}-\int_{S} U_{a}^{k}(\tilde{\boldsymbol{x}}, \boldsymbol{x}) p_{a}(\boldsymbol{x}) d S_{x}=0
$$

where the free-term is given by:

$$
c_{k a}(\tilde{\boldsymbol{x}})=\lim _{\varepsilon \rightarrow 0} \int_{s_{\varepsilon}} T_{a}^{k}(\tilde{\boldsymbol{x}}, \boldsymbol{x}) d S_{x} \quad\left(c_{k a}(\tilde{\boldsymbol{x}})=\frac{1}{2} \delta_{k a} \text { if } S \text { is smooth at } \tilde{\boldsymbol{x}}\right)
$$

and $f$ denotes integration in the Cauchy principal value $(\mathrm{CPV})$ sense. Recall that the choice of a spherical shape for $v_{\varepsilon}$ is convenient but by no means mandatory; this point has been discussed at length in the BEM literature [27].

A governing 'derivative BIE' for the material derivatives $\stackrel{\star}{\boldsymbol{u}}, \stackrel{\star}{\boldsymbol{p}}$ of the boundary variables can be sought by applying the material derivative formula (14) to identity (16) with finite $\varepsilon$. This process leads, as shown in Appendix A.2, to the identity:

$$
\begin{aligned}
& \int_{S_{\varepsilon}+s_{\varepsilon}}\left\{T_{a}^{k}(\tilde{\boldsymbol{x}}, \boldsymbol{x}) \stackrel{\star}{u}_{a}(\boldsymbol{x})-U_{a}^{k}(\tilde{\boldsymbol{x}}, \boldsymbol{x}) \stackrel{\star}{p}_{a}(\boldsymbol{x})\right\} d S_{x} \\
& =\int_{S_{\varepsilon}+s_{\varepsilon}}\left\{\Sigma_{a b}^{k}(\tilde{\boldsymbol{x}}, \boldsymbol{x}) e_{c b i} R_{i} u_{a}(\boldsymbol{x})-\rho \omega^{2} U_{a}^{k}(\tilde{\boldsymbol{x}}, \boldsymbol{x}) u_{a}(\boldsymbol{x}) n_{c}(\boldsymbol{x})\right\}\left[\theta_{c}(\boldsymbol{x})-\theta_{c}(\tilde{\boldsymbol{x}})\right] d S_{x} \\
& +\int_{S_{\varepsilon}+s_{\varepsilon}}\left\{U_{a, c}^{k}(\tilde{\boldsymbol{x}}, \boldsymbol{x})\left[\theta_{c}(\boldsymbol{x})-\theta_{c}(\tilde{\boldsymbol{x}})\right]+U_{a}^{k}(\tilde{\boldsymbol{x}}, \boldsymbol{x}) D_{c} \theta_{c}(\boldsymbol{x})\right\} p_{a}(\boldsymbol{x}) d S_{x}
\end{aligned}
$$

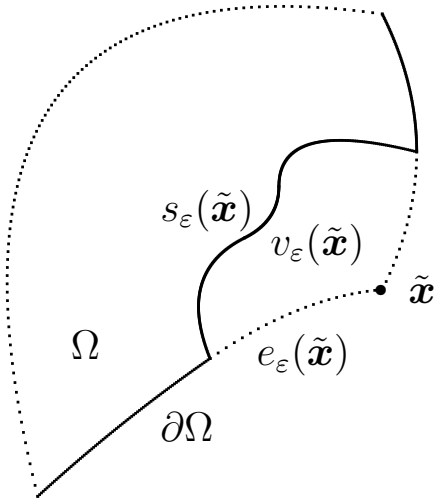

Figure 2: Exclusion of the singular point $\tilde{\boldsymbol{x}}$ by a vanishing neighbourhood $v_{\varepsilon}(\tilde{\boldsymbol{x}})$. 
where $f_{, c}$ denotes the Cartesian partial derivative with respect to the $c$-coordinate of the integration point $\boldsymbol{x}$ and $R_{i}$ is defined by (12). Then, the limiting form of this identity when $\varepsilon \rightarrow 0$ must be established. Assume that the material derivative $\stackrel{\star}{\boldsymbol{u}}$ and the transformation velocity $\boldsymbol{\theta}$ have respectively $C^{0, \alpha}$ and $C^{0,1}$ regularity at $\boldsymbol{x}=\tilde{\boldsymbol{x}}$. The limiting process $\varepsilon \rightarrow 0$ in the left-hand side then reproduces that leading to Eq. (18). Moreover, the integrals over $s_{\varepsilon}$ in the right-hand side vanish while those over $S_{\varepsilon}$ are convergent, weakly singular integrals. The derivative BIE obtained from Eq. (16) thus reads:

$$
\begin{aligned}
& c_{k a} \stackrel{\star}{u}_{a}(\tilde{\boldsymbol{x}})+f_{S} T_{a}^{k}(\tilde{\boldsymbol{x}}, \boldsymbol{x}) \stackrel{\star}{u}_{a}(\boldsymbol{x}) d S_{x}-\int_{S} U_{a}^{k}(\tilde{\boldsymbol{x}}, \boldsymbol{x}) \stackrel{\star}{p}_{a}(\boldsymbol{x}) d S_{x} \\
& =\int_{S}\left\{\Sigma_{a b}^{k}(\tilde{\boldsymbol{x}}, \boldsymbol{x}) e_{c b i} R_{i} u_{a}(\boldsymbol{x})-\rho \omega^{2} U_{a}^{k}(\tilde{\boldsymbol{x}}, \boldsymbol{x}) u_{a}(\boldsymbol{x}) n_{c}(\boldsymbol{x})\right\}\left[\theta_{c}(\boldsymbol{x})-\theta_{c}(\tilde{\boldsymbol{x}})\right] d S_{x} \\
& -\int_{S}\left\{U_{a, c}^{k}(\tilde{\boldsymbol{x}}, \boldsymbol{x})\left[\theta_{c}(\boldsymbol{x})-\theta_{c}(\tilde{\boldsymbol{x}})\right]+U_{a}^{k}(\tilde{\boldsymbol{x}}, \boldsymbol{x}) D_{c} \theta_{c}(\boldsymbol{x})\right\} p_{a}(\boldsymbol{x}) d S_{x}
\end{aligned}
$$

\subsection{Material derivative of hypersingular BIE}

Hypersingular BIE formulations arise in connection with the differentiation with respect to the source point coordinates $x_{\ell}$ of Eq. (16), i.e. as the limiting form when $\varepsilon \rightarrow 0$ of the exterior representation formula for the displacement gradient:

$$
\int_{S_{\varepsilon}+s_{\varepsilon}}\left\{\Sigma_{k \ell, \bar{b}}^{a}(\tilde{\boldsymbol{x}}, \boldsymbol{x}) n_{\ell}(\boldsymbol{x}) u_{k}(\boldsymbol{x})-U_{k, \bar{b}}^{a}(\tilde{\boldsymbol{x}}, \boldsymbol{x}) p_{k}(\boldsymbol{x})\right\} d S_{x}=0
$$

where a $C^{1, \alpha}$ regularity at $\boldsymbol{x}=\tilde{\boldsymbol{x}}$ is assumed for both $S$ and $\boldsymbol{u}$, implying $\boldsymbol{\sigma} \in C^{0, \alpha}$ (the overbar in $f_{, \bar{b}}$ indicates that the partial derivative is taken with respect to the $\ell$-coordinate of the collocation point $\tilde{\boldsymbol{x}})$. Performing the limiting process $\varepsilon \rightarrow 0$ and taking the inner product of the result with $C_{i j a b} n_{j}(\tilde{\boldsymbol{x}})$ in Eq. (22) yields the well-known hypersingular BIE (see e.g. Guiggiani [26]):

$$
\frac{1}{2} p_{i}(\tilde{\boldsymbol{x}})+f_{S} D_{i k}(\tilde{\boldsymbol{x}}, \boldsymbol{x}) u_{k}(\boldsymbol{x}) d S_{x}-f_{S} T_{i}^{k}(\boldsymbol{x}, \tilde{\boldsymbol{x}}) p_{k}(\boldsymbol{x}) d S_{x}=0
$$

having used the identity $C_{i j a b} U_{k, \bar{b}}^{a}(\tilde{\boldsymbol{x}}, \boldsymbol{x}) n_{j}(\tilde{\boldsymbol{x}})=T_{i}^{k}(\boldsymbol{x}, \tilde{\boldsymbol{x}})$, introduced the new kernel

$$
D_{i k}(\tilde{\boldsymbol{x}}, \boldsymbol{x})=C_{i j a b} \Sigma_{k \ell, \bar{b}}^{a}(\tilde{\boldsymbol{x}}, \boldsymbol{x}) n_{j}(\tilde{\boldsymbol{x}}) n_{\ell}(\boldsymbol{x})
$$

and where fdenotes the integration in the finite part (FP) sense, i.e.:

$$
f_{S} D_{i k}(\tilde{\boldsymbol{x}}, \boldsymbol{x}) u_{k}(\boldsymbol{x}) d S_{x} \equiv \lim _{\varepsilon \rightarrow 0}\left\{u_{k}(\tilde{\boldsymbol{x}}) \int_{s_{\varepsilon}} D_{i k}(\tilde{\boldsymbol{x}}, \boldsymbol{x}) d S_{x}+\int_{S_{\varepsilon}} D_{i k}(\tilde{\boldsymbol{x}}, \boldsymbol{x}) u_{k}(\boldsymbol{x}) d S_{x}\right\}
$$

A short proof of Eq. (23) is given for completeness in Appendix A.3.

Following the same path of reasoning as in section 4.1, the corresponding derivative BIE can be established by first applying the material derivative formulas (14), (15) to identity (22) with finite 
$\varepsilon$ :

$$
\begin{aligned}
\int_{S_{\varepsilon}+s_{\varepsilon}} & \left\{\Sigma_{k \ell, \bar{b}}^{a}(\tilde{\boldsymbol{x}}, \boldsymbol{x}) n_{\ell}(\boldsymbol{x}) \stackrel{\star}{u_{k}}(\boldsymbol{x})-U_{k, \bar{b}}^{a}(\tilde{\boldsymbol{x}}, \boldsymbol{x}) \stackrel{\star}{p}_{k}(\boldsymbol{x})\right\} d S_{x} \\
& +\int_{S_{\varepsilon}+s_{\varepsilon}} u_{k}(\boldsymbol{x})\left\{\Sigma_{k \ell, \bar{b} c}^{a}(\tilde{\boldsymbol{x}}, \boldsymbol{x})\left[\theta_{c}(\boldsymbol{x})-\theta_{c}(\tilde{\boldsymbol{x}})\right] n_{\ell}(\boldsymbol{x})+\Sigma_{k \ell, \bar{b}}^{a} e_{\ell c i} R_{i} \theta_{c}(\boldsymbol{x})\right\} d S_{x} \\
& -\int_{S_{\varepsilon}+s_{\varepsilon}}\left\{U_{k, \bar{b} c}^{a}(\tilde{\boldsymbol{x}}, \boldsymbol{x})\left[\theta_{c}(\boldsymbol{x})-\theta_{c}(\tilde{\boldsymbol{x}})\right]+U_{k, \bar{b}}^{a}(\tilde{\boldsymbol{x}}, \boldsymbol{x}) D_{c} \theta_{c}\right\} p_{k}(\boldsymbol{x}) d S_{x}=0
\end{aligned}
$$

This identity can be recast into the following equivalent form, as shown in Appendix A.4:

$$
\begin{aligned}
\int_{S_{\varepsilon}}\left\{\Sigma_{k \ell, \bar{b}}^{a}(\tilde{\boldsymbol{x}}, \boldsymbol{x}) n_{\ell}(\boldsymbol{x}) \stackrel{\star}{u_{k}}(\boldsymbol{x})-U_{k, \bar{b}}^{a}(\tilde{\boldsymbol{x}}, \boldsymbol{x}) \stackrel{\star}{p}_{k}(\boldsymbol{x})\right\} d S_{x} \\
\quad+\stackrel{\star}{u}_{k}(\tilde{\boldsymbol{x}}) \int_{s_{\varepsilon}} \Sigma_{k \ell, \bar{b}}^{a}(\tilde{\boldsymbol{x}}, \boldsymbol{x}) n_{\ell}(\boldsymbol{x}) d S_{x}+\left(u_{k, \bar{b}}\right)^{\star}(\tilde{\boldsymbol{x}}) \int_{s_{\varepsilon}} T_{k}^{a}(\tilde{\boldsymbol{x}}, \boldsymbol{x}) d S_{x} \\
\quad+\int_{S_{\varepsilon}}\left\{e_{c \ell d} R_{d} u_{k}(\boldsymbol{x}) \Sigma_{k \ell, \bar{b}}^{a}(\tilde{\boldsymbol{x}}, \boldsymbol{x})-\rho \omega^{2} u_{k}(\boldsymbol{x}) U_{k, \bar{b}}^{a}(\tilde{\boldsymbol{x}}, \boldsymbol{x}) n_{c}(\boldsymbol{x})\right\}\left[\theta_{c}(\boldsymbol{x})-\theta_{c}(\tilde{\boldsymbol{x}})\right] d S_{x} \\
-\int_{S_{\varepsilon}}\left\{U_{k, \bar{b} c}^{a}(\tilde{\boldsymbol{x}}, \boldsymbol{x})\left[\theta_{c}(\boldsymbol{x})-\theta_{c}(\tilde{\boldsymbol{x}})\right]+U_{k, \bar{b}}^{a}(\tilde{\boldsymbol{x}}, \boldsymbol{x}) D_{c} \theta_{c}\right\} p_{k}(\boldsymbol{x}) d S_{x} \\
\quad-e_{\ell b q}\left(u_{k, d}\right)^{\star}(\tilde{\boldsymbol{x}}) \int_{c_{\varepsilon}} \tau_{q}(\boldsymbol{x}) \Sigma_{k \ell}^{a}(\tilde{\boldsymbol{x}}, \boldsymbol{x})\left(y_{d}-x_{d}\right) d s_{x} \\
\quad+e_{\ell b q} \stackrel{\stackrel{\star}{\sigma}}{k \ell}(\tilde{\boldsymbol{x}}) \int_{c_{\varepsilon}} \tau_{q}(\boldsymbol{x}) U_{k}^{a}(\tilde{\boldsymbol{x}}, \boldsymbol{x}) d S_{x} \\
\quad+\sigma_{k \ell}(\tilde{\boldsymbol{x}}) e_{c \ell q} \int_{c_{\varepsilon}} \tau_{q}(\boldsymbol{x}) U_{k, \bar{b}}^{a}(\tilde{\boldsymbol{x}}, \boldsymbol{x})\left[\theta_{c}(\boldsymbol{x})-\theta_{c}(\tilde{\boldsymbol{x}})\right] d s_{x}+O(\varepsilon)=0
\end{aligned}
$$

This result relies on both $\stackrel{\star}{\boldsymbol{u}}$ and $\boldsymbol{\theta}$ having a $C^{1, \alpha}$ regularity at $\boldsymbol{x}=\tilde{\boldsymbol{x}}$, in addition to the already stated smoothness assumptions.

Now the limiting process $\varepsilon \rightarrow 0$ is carried out in the above identity, using again a spherical exclusion neighbourhood $v_{\varepsilon}$. Under this assumption, the last three contour integrals in Eq. (27) vanish in the limit $\varepsilon \rightarrow 0$, and Eq. (19) applies. The limiting process in the first two integrals reproduces that leading to Eq. (23). The remaining integrals over $S_{\varepsilon}$ produce new FP and CPV integrals over $S$. After taking the inner product of the result with $C_{i j a b} n_{j}(\tilde{\boldsymbol{x}})$, the resulting derivative BIE associated to the hypersingular BIE finally reads (putting $\left.T_{i, c}^{k}(\boldsymbol{x}, \tilde{\boldsymbol{x}})=\Sigma_{i j, c}^{k}(\boldsymbol{x}, \tilde{\boldsymbol{x}}) n_{j}(\tilde{\boldsymbol{x}})\right)$ :

$$
\begin{aligned}
& \frac{1}{2} \stackrel{\star}{\sigma}_{i j}(\tilde{\boldsymbol{x}}) n_{j}(\tilde{\boldsymbol{x}})+f_{S} D_{i k}(\tilde{\boldsymbol{x}}, \boldsymbol{x}) \stackrel{\star}{u}_{k}(\boldsymbol{x}) d S_{x}-f_{S} T_{i}^{k}(\boldsymbol{x}, \tilde{\boldsymbol{x}}) \stackrel{\star}{p}_{k}(\boldsymbol{x}) d S_{x} \\
& \quad+C_{i j a b} n_{j}(\tilde{\boldsymbol{x}}) f_{S}\left\{e_{c \ell d} R_{d} u_{k}(\boldsymbol{x}) \Sigma_{k \ell, \bar{b}}^{a}(\tilde{\boldsymbol{x}}, \boldsymbol{x})-\rho \omega^{2} u_{k}(\boldsymbol{x}) U_{k, \bar{b}}^{a}(\tilde{\boldsymbol{x}}, \boldsymbol{x}) n_{c}(\boldsymbol{x})\right\}\left[\theta_{c}(\boldsymbol{x})-\theta_{c}(\tilde{\boldsymbol{x}})\right] d S_{x} \\
& \quad-f_{S}\left\{T_{i, c}^{k}(\boldsymbol{x}, \tilde{\boldsymbol{x}})\left[\theta_{c}(\boldsymbol{x})-\theta_{c}(\tilde{\boldsymbol{x}})\right]+T_{i}^{k}(\boldsymbol{x}, \tilde{\boldsymbol{x}}) D_{c} \theta_{c}\right\} p_{k}(\boldsymbol{x}) d S_{x}=0
\end{aligned}
$$

\section{Collocation derivative BIEs for a cracked body}

The results of sections 4.1 and 4.2, which were established a priori for domains without cracks, can be extended to cracked bodies following a simple procedure. Indeed, introduce a partition of $\Omega$ into 
two fictitious subdomains $\Omega_{1}$ and $\Omega_{2}=\Omega \backslash \Omega_{1}$, where $\Omega_{1}$ is chosen such that (i) $\Omega_{1}$ is embedded in $\Omega$ and (ii) the boundary of $\Omega_{1}$ contains $\Gamma$. Writing the displacement integral equation (18) separately for the two subdomains (and with $\tilde{\boldsymbol{x}} \in S$ ) and adding them yields the strongly singular BIE:

$$
c_{k a}(\tilde{\boldsymbol{x}}) u_{a}(\tilde{\boldsymbol{x}})+f_{S} T_{a}^{k}(\tilde{\boldsymbol{x}}, \boldsymbol{x}) u_{a}(\boldsymbol{x}) d S_{x}-\int_{S} U_{a}^{k}(\tilde{\boldsymbol{x}}, \boldsymbol{x}) p_{a}(\boldsymbol{x}) d S_{x}-\int_{\Gamma} T_{a}^{k}(\tilde{\boldsymbol{x}}, \boldsymbol{x}) \phi_{a}(\boldsymbol{x}) d S_{x}=0
$$

A similar procedure applied to the hypersingular BIE (23) leads to the equation:

$$
\frac{1}{2} \hat{p}_{i}(\tilde{\boldsymbol{x}})+f_{S} D_{i k}(\tilde{\boldsymbol{x}}, \boldsymbol{x}) u_{k}(\boldsymbol{x}) d S_{x}-\int_{S} T_{i}^{k}(\boldsymbol{x}, \tilde{\boldsymbol{x}}) p_{k}(\boldsymbol{x}) d S_{x}-\int_{\Gamma} D_{i k}(\tilde{\boldsymbol{x}}, \boldsymbol{x}) \phi_{k}(\boldsymbol{x}) d S_{x}=0
$$

for $\tilde{\boldsymbol{x}} \in S_{p}$, and:

$$
\int_{S} D_{i k}(\tilde{\boldsymbol{x}}, \boldsymbol{x}) u_{k}(\boldsymbol{x}) d S_{x}-\int_{S} T_{i}^{k}(\boldsymbol{x}, \tilde{\boldsymbol{x}}) p_{k}(\boldsymbol{x}) d S_{x}-f_{\Gamma} D_{i k}(\tilde{\boldsymbol{x}}, \boldsymbol{x}) \phi_{k}(\boldsymbol{x}) d S_{x}=0
$$

for $\tilde{\boldsymbol{x}} \in \Gamma$, the crack faces being assumed traction-free.

The collocation BIE approach for cracked solids (using the displacement discontinuity method) consists in coupling the displacement BIE (29) for collocation points on $S$ and the traction BIE (31) for collocation points on $\Gamma$. The dual collocation BIE formulation could of course be obtained following a similar approach. Taking into account the boundary conditions (2) and the corresponding partition of $S$, the resulting coupled system of integral equations has the structure:

$$
\left\{\begin{array}{l}
\mathcal{H}_{p}(\boldsymbol{p})+\mathcal{G}_{u}(\boldsymbol{u})+\mathcal{G}_{\phi}(\phi)=-\mathcal{H}_{u}(\hat{\boldsymbol{p}})-\mathcal{G}_{p}(\hat{\boldsymbol{u}}) \\
\mathcal{S}_{p}(\boldsymbol{p})+\mathcal{D}_{u}(\boldsymbol{u})+\mathcal{D}_{\phi}(\phi)=-\mathcal{S}_{u}(\hat{\boldsymbol{p}})-\mathcal{D}_{p}(\hat{\boldsymbol{u}})
\end{array}\right.
$$

where $(\hat{\boldsymbol{p}}, \hat{\boldsymbol{u}})$ are the prescribed tractions (on $\left.S_{p}\right)$ and displacements (on $\left.S_{u}\right) ;(\boldsymbol{p}, \boldsymbol{u}, \boldsymbol{\phi})$ are the unknown tractions (on $S_{u}$ ), displacements $\left(\right.$ on $S_{p}$ ) and crack opening displacement (on $\left.\Gamma\right) ;(\mathcal{G}, \mathcal{H})$ and $(\mathcal{S}, \mathcal{D})$ denote the pairs of linear integral operators (including the free-term) acting on tractions and displacements in Eqs. (29) and (31), respectively; the indices $p, u, \phi$ indicate that the corresponding integral operator acts on functions defined on the geometrical support of $(\boldsymbol{p}, \boldsymbol{u}, \boldsymbol{\phi})$, i.e. on $S_{u}, S_{p}, \Gamma$, respectively. Eq. (32) refers to either the continuous system of integral equations in symbolic (operator) notation or the set of BEM discretized linear equations.

The derivative BIEs for the cracked solid are obtained in the same manner from Eqs. (21) and (28). Assuming that the external boundary $S$ is not affected by the geometrical transformation, the displacement derivative BIE (21) becomes (with $\tilde{\boldsymbol{x}} \in S$ )):

$$
\begin{array}{r}
c_{k a}(\tilde{\boldsymbol{x}}) \stackrel{\star}{\mathrm{u}_{a}}(\tilde{\boldsymbol{x}})+f_{S} T_{a}^{k}(\tilde{\boldsymbol{x}}, \boldsymbol{x}) \stackrel{\star}{\mathrm{u}_{a}}(\boldsymbol{x}) d S_{x}-\int_{S} U_{a}^{k}(\tilde{\boldsymbol{x}}, \boldsymbol{x}) \stackrel{\star}{p}_{a}(\boldsymbol{x}) d S_{x}-\int_{\Gamma} T_{a}^{k}(\tilde{\boldsymbol{x}}, \boldsymbol{x}) \stackrel{\star}{\phi}(\boldsymbol{x}) d S_{x} \\
=\int_{\Gamma}\left\{\Sigma_{a b}^{k}(\tilde{\boldsymbol{x}}, \boldsymbol{x}) e_{c b i} R_{i} \phi_{a}(\boldsymbol{x})-\rho \omega^{2} U_{a}^{k}(\tilde{\boldsymbol{x}}, \boldsymbol{x}) \phi_{a}(\boldsymbol{x}) n_{c}(\boldsymbol{x})\right\} \theta_{c}(\boldsymbol{x}) d S_{x}
\end{array}
$$


The traction derivative BIE (28) becomes:

$$
\begin{aligned}
& -\int_{\Gamma} D_{i k}(\tilde{\boldsymbol{x}}, \boldsymbol{x}) \stackrel{\star}{\phi_{k}}(\boldsymbol{x}) d S_{x}+f_{S} D_{i k}(\tilde{\boldsymbol{x}}, \boldsymbol{x}) \stackrel{\star}{u_{k}}(\boldsymbol{x}) d S_{x}-f_{S} T_{i}^{k}(\boldsymbol{x}, \tilde{\boldsymbol{x}}) \stackrel{\star}{p_{k}}(\boldsymbol{x}) d S_{x} \\
& -C_{i j a b} n_{j}(\tilde{\boldsymbol{x}}) \int_{\Gamma}\left\{e_{c \ell d} R_{d} u_{k}(\boldsymbol{x}) \Sigma_{k \ell, \bar{b}}^{a}(\tilde{\boldsymbol{x}}, \boldsymbol{x})-\rho \omega^{2} u_{k}(\boldsymbol{x}) U_{k, \bar{b}}^{a}(\tilde{\boldsymbol{x}}, \boldsymbol{x}) n_{c}(\boldsymbol{x})\right\} \theta_{c}(\boldsymbol{x}) d S_{x}=0
\end{aligned}
$$

for $\tilde{\boldsymbol{x}} \in S_{p}$ (using that $\stackrel{\star}{\hat{\boldsymbol{p}}}=\mathbf{0}, \boldsymbol{\theta}=\mathbf{0}$ and $\stackrel{\star}{\boldsymbol{n}}=\mathbf{0}$ on $S_{p}$ ). Similarly, if $\tilde{\boldsymbol{x}} \in \Gamma$, Eq (28) becomes:

$$
\begin{aligned}
\frac{1}{2}\left(\stackrel{\star}{\sigma}_{i j}\right. & \left.\left(\tilde{\boldsymbol{x}}^{+}\right)+\stackrel{\star}{\sigma}_{i j}\left(\tilde{\boldsymbol{x}}^{-}\right)\right) n_{j}(\tilde{\boldsymbol{x}})-f_{\Gamma} D_{i k}(\tilde{\boldsymbol{x}}, \boldsymbol{x}) \stackrel{\star}{\phi}_{k}(\boldsymbol{x}) d S_{x} \\
& +\int_{S} D_{i k}(\tilde{\boldsymbol{x}}, \boldsymbol{x}) \stackrel{\star}{u}_{k}(\boldsymbol{x}) d S_{x}-\int_{S} T_{i}^{k}(\boldsymbol{x}, \tilde{\boldsymbol{x}}) \stackrel{\star}{p}_{k}(\boldsymbol{x}) d S_{x} \\
& -C_{i j a b} n_{j}(\tilde{\boldsymbol{x}}) f_{\Gamma}\left\{e_{c \ell d} R_{d} u_{k}(\boldsymbol{x}) \Sigma_{k \ell, \bar{b}}^{a}(\tilde{\boldsymbol{x}}, \boldsymbol{x})-\rho \omega^{2} u_{k}(\boldsymbol{x}) U_{k, \bar{b}}^{a}(\tilde{\boldsymbol{x}}, \boldsymbol{x}) n_{c}(\boldsymbol{x})\right\}\left[\theta_{c}(\boldsymbol{x})-\theta_{c}(\tilde{\boldsymbol{x}})\right] d S_{x} \\
& -\theta_{c}(\tilde{\boldsymbol{x}}) \int_{S}\left\{e_{\ell c d} C_{i j a b} n_{j}(\tilde{\boldsymbol{x}}) u_{k}(\boldsymbol{x}) \Sigma_{k \ell, \bar{b} c}^{a}(\tilde{\boldsymbol{x}}, \boldsymbol{x}) n_{\ell}(\boldsymbol{x})-T_{i, c}^{k}(\boldsymbol{x}, \tilde{\boldsymbol{x}}) p_{k}(\boldsymbol{x})\right\} d S_{x}=0
\end{aligned}
$$

Besides, the free surface condition on the crack faces must be maintained in any crack perturbation, which implies:

$$
\stackrel{\star}{\sigma}_{i j}\left(\tilde{\boldsymbol{x}}^{ \pm}\right) n_{j}(\tilde{\boldsymbol{x}})=-\sigma_{i j}\left(\tilde{\boldsymbol{x}}^{ \pm}\right) \stackrel{\star}{n}_{j}(\tilde{\boldsymbol{x}})
$$

and $\sigma_{i j}\left(\tilde{\boldsymbol{x}}^{ \pm}\right)$can be evaluated using the hypersingular stress representation formula.

The derivative integral equations associated with the usual collocation method are the derivative displacement BIE (33) for collocation points on $S$ and the derivative traction BIE (35-36) for collocation points on $\Gamma$. The resulting coupled system of integral equations has the structure:

$$
\left\{\begin{aligned}
& \mathcal{H}_{p}(\stackrel{\star}{\boldsymbol{p}})+\mathcal{G}_{u}(\stackrel{\star}{\boldsymbol{u}})+\mathcal{G}_{\phi}(\stackrel{\star}{\boldsymbol{\phi}})=-\mathcal{G}_{\phi}^{\star}(\boldsymbol{\phi} ; \boldsymbol{\theta}) \\
& \mathcal{S}_{p}(\stackrel{\star}{\boldsymbol{p}})+\mathcal{D}_{u}(\stackrel{\star}{\boldsymbol{u}})+\mathcal{D}_{\phi}(\stackrel{\star}{\boldsymbol{\phi}})=-\mathcal{S}_{p}^{\star}(\boldsymbol{p} ; \boldsymbol{\theta})-\mathcal{D}_{u}^{\star}(\boldsymbol{u} ; \boldsymbol{\theta})-\mathcal{D}_{\phi}^{\star}(\boldsymbol{\phi} ; \boldsymbol{\theta}) \\
& \quad-\mathcal{S}_{u}^{\star}(\hat{\boldsymbol{p}} ; \boldsymbol{\theta})-\mathcal{D}_{p}^{\star}(\hat{\boldsymbol{u}} ; \boldsymbol{\theta})-\mathcal{S}_{u}(\stackrel{\star}{\hat{\boldsymbol{p}}})-\mathcal{D}_{p}(\stackrel{\star}{\hat{\boldsymbol{u}}})
\end{aligned}\right.
$$

The blocks $\mathcal{S}_{p}^{\star}(\boldsymbol{\theta}), \mathcal{S}_{u}^{\star}(\boldsymbol{\theta}), \mathcal{G}_{\phi}^{\star}(\boldsymbol{\theta}), \mathcal{D}_{p}^{\star}(\boldsymbol{\theta}), \mathcal{D}_{u}^{\star}(\boldsymbol{\theta})$ and $\mathcal{D}_{\phi}^{\star}(\boldsymbol{\theta})$ depend linearly on $\boldsymbol{\theta}$. A set of derivative equations could be established for the dual BIE formulation as well.

The displacement BIE and its material derivative BIE can be employed alone (i.e without invoking a traction BIE) for crack problems using the multiregion approach: the cracked domain $\Omega$ is split into two fictitious subdomains $\Omega_{1}, \Omega_{2}$ such that $\Gamma$ belongs to the fictitious interface separating $\Omega_{1}$ and $\Omega_{2}$. Thus, avoiding a hypersingular BIE requires the coupling of two BIE formulations (except when the crack $\Gamma$ lies on a symmetry plane of $\Omega$ ) and the introduction of supplementary degrees of freedom on the additional fictitious interface. 


\section{Symmetric Galerkin derivative BIEs for a cracked body}

\subsection{Symmetric Galerkin BIE formulation}

The symmetric Galerkin elastodynamic BIE governing formulation for a cracked solid can be obtained as follows (see e.g. [6] and the review [12] for a more complete account of SGBEMs):

- Equation (29) is multiplied by a trial traction $\boldsymbol{q}(\tilde{\boldsymbol{x}})$ and integrated over $\tilde{\boldsymbol{x}} \in S_{u}$;

- Equation (30) is multiplied by a trial displacement $\boldsymbol{v}(\tilde{\boldsymbol{x}})$ and integrated over $\tilde{\boldsymbol{x}} \in S_{p}$;

- Equation (31) is multiplied by a trial crack opening displacement $\boldsymbol{\psi}(\tilde{\boldsymbol{x}})$ and integrated over $\tilde{\boldsymbol{x}} \in \Gamma$.

This results in a symmetric system of governing equations on $(\boldsymbol{p}, \boldsymbol{u}, \boldsymbol{\phi})$ :

$$
\begin{aligned}
\mathcal{B}_{p p}(\boldsymbol{p}, \boldsymbol{q})+\mathcal{B}_{u p}(\boldsymbol{u}, \boldsymbol{q})+\mathcal{B}_{\phi p}(\boldsymbol{\phi}, \boldsymbol{q})=\mathcal{F}_{u p}(\hat{\boldsymbol{u}}, \boldsymbol{q})+\mathcal{F}_{p p}(\hat{\boldsymbol{p}}, \boldsymbol{q}) \\
\mathcal{B}_{p u}(\boldsymbol{p}, \boldsymbol{v})+\mathcal{B}_{u u}(\boldsymbol{u}, \boldsymbol{v})+\mathcal{B}_{\phi u}(\boldsymbol{\phi}, \boldsymbol{v})=\mathcal{F}_{u u}(\hat{\boldsymbol{u}}, \boldsymbol{v})+\mathcal{F}_{p u}(\hat{\boldsymbol{p}}, \boldsymbol{v}) \\
\mathcal{B}_{p \phi}(\boldsymbol{p}, \boldsymbol{\psi})+\mathcal{B}_{u \phi}(\boldsymbol{u}, \boldsymbol{\psi})+\mathcal{B}_{\phi \phi}(\boldsymbol{\phi}, \boldsymbol{\psi})=\mathcal{F}_{u \phi}(\hat{\boldsymbol{u}}, \boldsymbol{\psi})+\mathcal{F}_{p \phi}(\hat{\boldsymbol{p}}, \boldsymbol{\psi}) \\
\quad \text { with } \boldsymbol{q} \in H^{-1 / 2}\left(S_{u}\right), \boldsymbol{v} \in H_{0}^{1 / 2}\left(S_{p}\right), \boldsymbol{\psi} \in H_{0}^{1 / 2}(\Gamma) \text { and } \boldsymbol{v}=\mathbf{0} \text { on } \partial S_{p}, \boldsymbol{\psi}=\mathbf{0} \text { on } \partial \Gamma
\end{aligned}
$$

in terms of the bilinear forms:

$$
\begin{aligned}
\mathcal{B}_{p p}(\boldsymbol{p}, \boldsymbol{q}) & =-\int_{S_{u}} \int_{S_{u}} U_{a}^{k}(\tilde{\boldsymbol{x}}, \boldsymbol{x}) p_{a}(\boldsymbol{x}) q_{k}(\tilde{\boldsymbol{x}}) d S_{x} d S_{\tilde{x}} \\
\mathcal{B}_{u u}(\boldsymbol{u}, \boldsymbol{v}) & =-\int_{S_{p}} \int_{S_{p}}\left\{B_{i k q s}(\tilde{\boldsymbol{x}}, \boldsymbol{x}) R_{s} u_{k}(\boldsymbol{x}) R_{q} v_{i}(\tilde{\boldsymbol{x}})+A_{i k}(\tilde{\boldsymbol{x}}, \boldsymbol{x}) u_{k}(\boldsymbol{x}) v_{i}(\tilde{\boldsymbol{x}})\right\} d S_{x} d S_{\tilde{x}} \\
\mathcal{B}_{\phi \phi}(\boldsymbol{\phi}, \boldsymbol{\psi}) & =-\int_{\Gamma} \int_{\Gamma}\left\{B_{i k q s}(\tilde{\boldsymbol{x}}, \boldsymbol{x}) R_{s} \phi_{k}(\boldsymbol{x}) R_{q} \psi_{i}(\tilde{\boldsymbol{x}})+A_{i k}(\tilde{\boldsymbol{x}}, \boldsymbol{x}) \phi_{k}(\boldsymbol{x}) \psi_{i}(\tilde{\boldsymbol{x}})\right\} d S_{x} d S_{\tilde{x}}
\end{aligned}
$$

which are symmetric, and

$$
\begin{aligned}
\mathcal{B}_{u p}(\boldsymbol{u}, \boldsymbol{q}) & =\int_{S_{u}} \int_{S_{p}} T_{a}^{k}(\tilde{\boldsymbol{x}}, \boldsymbol{x}) u_{a}(\boldsymbol{x}) q_{k}(\tilde{\boldsymbol{x}}) d S_{x} d S_{\tilde{x}}=\mathcal{B}_{p u}(\boldsymbol{q}, \boldsymbol{u}) \\
\mathcal{B}_{\phi p}(\boldsymbol{\phi}, \boldsymbol{q}) & =-\int_{S_{u}} \int_{\Gamma} T_{a}^{k}(\tilde{\boldsymbol{x}}, \boldsymbol{x}) \phi_{a}(\boldsymbol{x}) q_{k}(\tilde{\boldsymbol{x}}) d S_{x} d S_{\tilde{x}}=\mathcal{B}_{p \phi}(\boldsymbol{q}, \boldsymbol{\phi}) \\
\mathcal{B}_{u \phi}(\boldsymbol{u}, \boldsymbol{\psi}) & =\int_{\Gamma} \int_{S_{p}} \psi_{i}(\tilde{\boldsymbol{x}}) D_{i k}(\tilde{\boldsymbol{x}}, \boldsymbol{x}) u_{k}(\boldsymbol{x}) d S_{x} d S_{\tilde{x}}=\mathcal{B}_{\phi u}(\boldsymbol{\psi}, \boldsymbol{u})
\end{aligned}
$$


and the linear forms:

$$
\begin{aligned}
\mathcal{F}_{u p}(\hat{\boldsymbol{u}}, \boldsymbol{q}) & =-\frac{1}{2} \int_{S_{u}} \hat{u}_{a}(\tilde{\boldsymbol{x}}) q_{a}(\tilde{\boldsymbol{x}}) d S_{\tilde{x}}-\int_{S_{u}} q_{k}(\tilde{\boldsymbol{x}}) f_{S_{u}} T_{a}^{k}(\tilde{\boldsymbol{x}}, \boldsymbol{x}) \hat{u}_{a}(\boldsymbol{x}) d S_{x} d S_{\tilde{x}} \\
\mathcal{F}_{p p}(\hat{\boldsymbol{p}}, \boldsymbol{q}) & =\int_{S_{u}} \int_{S_{p}} q_{k}(\tilde{\boldsymbol{x}}) U_{a}^{k}(\tilde{\boldsymbol{x}}, \boldsymbol{x}) \hat{p}_{a}(\boldsymbol{x}) d S_{x} d S_{\tilde{x}} \\
\mathcal{F}_{u u}(\hat{\boldsymbol{u}}, \boldsymbol{v}) & =\int_{S_{p}} \int_{S_{u}}\left\{B_{i k q s}(\tilde{\boldsymbol{x}}, \boldsymbol{x}) R_{s} \hat{u}_{k}(\boldsymbol{x}) R_{q} v_{i}(\tilde{\boldsymbol{x}})+A_{i k}(\tilde{\boldsymbol{x}}, \boldsymbol{x}) \hat{u}_{k}(\boldsymbol{x}) v_{i}(\tilde{\boldsymbol{x}})\right\} d S_{x} d S_{\tilde{x}} \\
\mathcal{F}_{p u}(\hat{\boldsymbol{p}}, \boldsymbol{v}) & =\frac{1}{2} \int_{S_{p}} \hat{p}_{i}(\tilde{\boldsymbol{x}}) v_{i}(\tilde{\boldsymbol{x}}) d S_{\tilde{x}}-\int_{S_{p}} \hat{p}_{a}(\boldsymbol{x}) f_{S_{p}} T_{i}^{k}(\boldsymbol{x}, \tilde{\boldsymbol{x}}) v_{i}(\tilde{\boldsymbol{x}}) d S_{x} d S_{\tilde{x}} \\
\mathcal{F}_{u \phi}(\hat{\boldsymbol{u}}, \boldsymbol{\psi}) & =-\int_{\Gamma} \int_{S_{u}} \psi_{i}(\tilde{\boldsymbol{x}}) D_{i k}(\tilde{\boldsymbol{x}}, \boldsymbol{x}) \hat{u}_{k}(\boldsymbol{x}) d S_{x} d S_{\tilde{x}} \\
\mathcal{F}_{p \phi}(\hat{\boldsymbol{p}}, \boldsymbol{\psi}) & =\int_{\Gamma} \int_{S_{p}} \psi_{i}(\tilde{\boldsymbol{x}}) T_{i}^{k}(\boldsymbol{x}, \tilde{\boldsymbol{x}}) \hat{p}_{a}(\boldsymbol{x}) d S_{x} d S_{\tilde{x}}
\end{aligned}
$$

Equations (40) and (47) together rely on the identity

$$
\begin{aligned}
\int_{S} v_{i}(\tilde{\boldsymbol{x}}) f_{S} D_{i k}(\tilde{\boldsymbol{x}}, \boldsymbol{x}) & u_{k}(\boldsymbol{x}) d S_{x} d S_{\tilde{x}} \\
& =\int_{S} \int_{S}\left\{B_{i k q s}(\tilde{\boldsymbol{x}}, \boldsymbol{x}) R_{s} u_{k}(\boldsymbol{x}) R_{q} v_{i}(\tilde{\boldsymbol{x}})+A_{i k}(\tilde{\boldsymbol{x}}, \boldsymbol{x}) u_{k}(\boldsymbol{x}) v_{i}(\tilde{\boldsymbol{x}})\right\} d S_{x} d S_{\tilde{x}}
\end{aligned}
$$

where $R_{i} f$ is defined by (12). The new kernels $B_{i k q s}(\tilde{\boldsymbol{x}}, \boldsymbol{x})$ and $A_{i k}(\tilde{\boldsymbol{x}}, \boldsymbol{x})$, given by Eqs. (88), (89), are associated with the decomposition (87) of the kernel $D_{i k}(\tilde{\boldsymbol{x}}, \boldsymbol{x})$ (given in [38] and generalized to anisotropic elasticity in [3]), by virtue of which the Stokes formula (13) could be applied. An identity similar to (51), with $S, \boldsymbol{u}, \boldsymbol{v}$ replaced with $\Gamma, \boldsymbol{\phi}, \boldsymbol{\psi}$, has been used to obtain (41).

\subsection{Symmetric Galerkin derivative BIEs}

The derivative BIEs in symmetric Galerkin form can be established in a straightforward fashion by calculating the material derivative of the set of symmetric Galerkin equations (38). Before giving detailed expressions, it is useful to point out that the material derivative of generic bilinear and linear operators $\mathcal{B}(\boldsymbol{a}, \boldsymbol{b})$ and $\mathcal{F}(\hat{\boldsymbol{a}}, \boldsymbol{b})$ (where $\boldsymbol{a}, \hat{\boldsymbol{a}}, \boldsymbol{b}$ denote unknown, prescribed and trial functions, respectively) must have the following form:

$$
\begin{aligned}
{[\mathcal{B}(\boldsymbol{a}, \boldsymbol{b})]^{\star} } & =\mathcal{B}(\stackrel{\star}{\boldsymbol{a}}, \boldsymbol{b})+\mathcal{B}^{\star}(\boldsymbol{a}, \boldsymbol{b} ; \boldsymbol{\theta}) \\
{[\mathcal{F}(\hat{\boldsymbol{a}}, \boldsymbol{b})]^{\star} } & =\mathcal{F}(\hat{\boldsymbol{a}}, \boldsymbol{b})+\mathcal{F}^{\star}(\hat{\boldsymbol{a}}, \boldsymbol{b} ; \boldsymbol{\theta})
\end{aligned}
$$

having omitted the terms involving the derivatives $\stackrel{\star}{b}$ of the test functions (they merely repeat the initial set of equations (38), so that $\stackrel{\star}{\boldsymbol{b}}=\mathbf{0}$ can be assumed without loss of generality); the new operators $\mathcal{B}^{\star}$ and $\mathcal{F}^{\star}$ depend linearly on $\boldsymbol{\theta}$. In particular, the governing bilinear operators for $(\boldsymbol{p}, \boldsymbol{u}, \boldsymbol{\phi})$ in $(38)$ and for $(\stackrel{\star}{\boldsymbol{p}}, \stackrel{\star}{\boldsymbol{u}}, \stackrel{\star}{\boldsymbol{\phi}})$ in the derivative symmetric Galerkin equations are necessarily the same. 
Moreover, the operators $\mathcal{B}_{p p}, \mathcal{B}_{\text {uu }}, \mathcal{B}_{\text {up }}$, Eqs. $(39,40,42)$ and $\mathcal{F}_{\text {up }}, \mathcal{F}_{p p}, \mathcal{F}_{\text {uu }}, \mathcal{F}_{\text {pu }}$, Eqs. $(45,46,47,48)$ are defined only in terms of integrals over $S_{u}$ and $S_{p}$, so that

$$
\mathcal{B}_{p p}^{\star}(\boldsymbol{p}, \boldsymbol{q} ; \boldsymbol{\theta})=\mathcal{B}_{u u}^{\star}(\boldsymbol{u}, \boldsymbol{v} ; \boldsymbol{\theta})=\mathcal{B}_{u p}^{\star}(\boldsymbol{u}, \boldsymbol{q} ; \boldsymbol{\theta})=\mathcal{B}_{p u}^{\star}(\boldsymbol{p}, \boldsymbol{v} ; \boldsymbol{\theta})=0
$$

and

$$
\mathcal{F}_{u p}^{\star}(\hat{\boldsymbol{u}}, \boldsymbol{q} ; \boldsymbol{\theta})=\mathcal{F}_{p p}^{\star}(\hat{\boldsymbol{p}}, \boldsymbol{q} ; \boldsymbol{\theta})=\mathcal{F}_{u u}^{\star}(\hat{\boldsymbol{u}}, \boldsymbol{v} ; \boldsymbol{\theta})=\mathcal{F}_{p u}^{\star}(\hat{\boldsymbol{p}}, \boldsymbol{v} ; \boldsymbol{\theta})=0
$$

The set of derivative symmetric Galerkin equations for $(\stackrel{\star}{\boldsymbol{p}}, \stackrel{\star}{\boldsymbol{u}}, \stackrel{\star}{\phi})$ thus has the form:

$$
\left\{\begin{aligned}
\mathcal{B}_{p p}(\stackrel{\star}{\boldsymbol{p}}, \boldsymbol{q})+\mathcal{B}_{u p}(\stackrel{\star}{\boldsymbol{u}}, \boldsymbol{q})+\mathcal{B}_{\phi p}(\stackrel{\star}{\boldsymbol{\phi}}, \boldsymbol{q})= & \mathcal{F}_{u p}(\stackrel{\star}{\hat{\boldsymbol{u}}}, \boldsymbol{q})+\mathcal{F}_{p p}(\stackrel{\star}{\hat{\boldsymbol{p}}}, \boldsymbol{q})-\mathcal{B}_{\phi p}^{\star}(\boldsymbol{\phi}, \boldsymbol{q} ; \boldsymbol{\theta}) \\
\mathcal{B}_{p u}(\stackrel{\star}{\boldsymbol{p}}, \boldsymbol{v})+\mathcal{B}_{u u}(\stackrel{\star}{\boldsymbol{u}}, \boldsymbol{v})+\mathcal{B}_{\phi u}(\stackrel{\star}{\boldsymbol{\phi}}, \boldsymbol{v})= & \mathcal{F}_{u u}(\stackrel{\star}{\hat{\boldsymbol{u}}}, \boldsymbol{q})+\mathcal{F}_{p u}(\stackrel{\star}{\hat{\boldsymbol{p}}}, \boldsymbol{q})-\mathcal{B}_{\phi u}^{\star}(\boldsymbol{\phi}, \boldsymbol{v} ; \boldsymbol{\theta}) \\
\mathcal{B}_{p \phi}(\stackrel{\star}{\boldsymbol{p}}, \boldsymbol{\psi})+\mathcal{B}_{u \phi}(\stackrel{\star}{\boldsymbol{u}}, \boldsymbol{\psi})+\mathcal{B}_{\phi \phi}(\stackrel{\star}{\boldsymbol{\phi}}, \boldsymbol{\psi})= & \mathcal{F}_{u \phi}(\stackrel{\star}{\boldsymbol{u}}, \boldsymbol{q})+\mathcal{F}_{p \phi}(\stackrel{\star}{\hat{\boldsymbol{p}}}, \boldsymbol{q})+\mathcal{F}_{u \phi}^{\star}(\hat{\boldsymbol{u}}, \boldsymbol{\psi} ; \boldsymbol{\theta})+\mathcal{F}_{p \phi}^{\star}(\hat{\boldsymbol{p}}, \boldsymbol{\psi} ; \boldsymbol{\theta}) \\
& -\mathcal{B}_{p \phi}^{\star}(\boldsymbol{p}, \boldsymbol{\psi} ; \boldsymbol{\theta})+\mathcal{B}_{u \phi}^{\star}(\boldsymbol{u}, \boldsymbol{\psi} ; \boldsymbol{\theta})+\mathcal{B}_{\phi \phi}^{\star}(\boldsymbol{\phi}, \boldsymbol{\psi} ; \boldsymbol{\theta})
\end{aligned}\right.
$$

with $\boldsymbol{q} \in H^{-1 / 2}\left(S_{u}\right), \boldsymbol{v} \in H_{0}^{1 / 2}\left(S_{p}\right), \boldsymbol{\psi} \in H_{0}^{1 / 2}(\Gamma)$ and $\boldsymbol{v}=\mathbf{0}$ on $\partial S_{p}, \boldsymbol{\psi}=\mathbf{0}$ on $\partial \Gamma$

where the various new operators appearing in the right-hand sides are found to be given by:

$$
\begin{aligned}
& \mathcal{B}_{\phi p}^{\star}(\boldsymbol{\phi}, \boldsymbol{q} ; \boldsymbol{\theta})=\int_{S_{p}} \int_{\Gamma} \Sigma_{a b}^{k}(\tilde{\boldsymbol{x}}, \boldsymbol{x}) e_{b c i} R_{i} \phi_{a}(\boldsymbol{x}) \theta_{c}(\boldsymbol{x}) q_{k}(\tilde{\boldsymbol{x}}) d S_{x} d S_{\tilde{x}} \\
& +\rho \omega^{2} \int_{S_{p}} \int_{\Gamma} U_{a}^{k}(\tilde{\boldsymbol{x}}, \boldsymbol{x}) \phi_{a}(\boldsymbol{x}) n_{c}(\boldsymbol{x}) \theta_{c}(\boldsymbol{x}) q_{k}(\tilde{\boldsymbol{x}}) d S_{x} d S_{\tilde{x}} \\
& \mathcal{B}_{\phi u}^{\star}(\boldsymbol{\phi}, \boldsymbol{v} ; \boldsymbol{\theta})=e_{c \ell d} \int_{S_{p}} \int_{\Gamma} C_{i j a b} n_{j}(\tilde{\boldsymbol{x}}) \Sigma_{k \ell, \bar{b}}^{a}(\tilde{\boldsymbol{x}}, \boldsymbol{x}) \theta_{c}(\boldsymbol{x}) R_{d} \phi_{k}(\boldsymbol{x}) v_{i}(\tilde{\boldsymbol{x}}) d S_{x} d S_{\tilde{x}} \\
& -\rho \omega^{2} \int_{S_{p}} \int_{\Gamma} T_{i}^{k}(\boldsymbol{x}, \tilde{\boldsymbol{x}}) n_{c}(\boldsymbol{x}) \theta_{c}(\boldsymbol{x}) \phi_{k}(\boldsymbol{x}) v_{i}(\tilde{\boldsymbol{x}}) d S_{x} d S_{\tilde{x}} \\
& \mathcal{B}_{p \phi}^{\star}(\boldsymbol{p}, \boldsymbol{\psi} ; \boldsymbol{\theta})=\mathcal{B}_{\phi p}^{\star}(\boldsymbol{\psi}, \boldsymbol{p} ; \boldsymbol{\theta}) \\
& \mathcal{B}_{u \phi}^{\star}(\boldsymbol{u}, \boldsymbol{\psi} ; \boldsymbol{\theta})=\mathcal{B}_{\phi u}^{\star}(\boldsymbol{\psi}, \boldsymbol{u} ; \boldsymbol{\theta}) \\
& \mathcal{B}_{\phi \phi}^{\star}(\boldsymbol{\phi}, \boldsymbol{\psi} ; \boldsymbol{\theta})=\int_{\Gamma} \int_{\Gamma} B_{i k q s}^{\star}(\tilde{\boldsymbol{x}}, \boldsymbol{x} ; \boldsymbol{\theta}) R_{s} \phi_{k}(\boldsymbol{x}) R_{q} \psi_{i}(\tilde{\boldsymbol{x}}) d S_{x} d S_{\tilde{x}} \\
& +\int_{\Gamma} \int_{\Gamma} A_{i k}^{\star}(\tilde{\boldsymbol{x}}, \boldsymbol{x} ; \boldsymbol{\theta}) \phi_{k}(\boldsymbol{x}) \psi_{i}(\tilde{\boldsymbol{x}}) d S_{x} d S_{\tilde{x}} \\
& \mathcal{F}_{u \phi}^{\star}(\hat{\boldsymbol{u}}, \boldsymbol{\psi} ; \boldsymbol{\theta})=e_{\ell c d} \int_{S_{u}} \int_{\Gamma} C_{i j a b} n_{j}(\tilde{\boldsymbol{x}}) \Sigma_{k \ell, \bar{b}}^{a}(\tilde{\boldsymbol{x}}, \boldsymbol{x}) \theta_{c}(\boldsymbol{x}) R_{d} \hat{u}_{k}(\boldsymbol{x}) \psi_{i}(\tilde{\boldsymbol{x}}) d S_{x} d S_{\tilde{x}} \\
& +\rho \omega^{2} \int_{S_{u}} \int_{\Gamma} T_{i}^{k}(\boldsymbol{x}, \tilde{\boldsymbol{x}}) n_{c}(\boldsymbol{x}) \theta_{c}(\boldsymbol{x}) \hat{u}_{k}(\boldsymbol{x}) \psi_{i}(\tilde{\boldsymbol{x}}) d S_{x} d S_{\tilde{x}} \\
& \mathcal{F}_{p \phi}^{\star}(\hat{\boldsymbol{p}}, \boldsymbol{\psi} ; \boldsymbol{\theta})=e_{c b i} \int_{S_{p}} \int_{\Gamma} \Sigma_{a b}^{k}(\tilde{\boldsymbol{x}}, \boldsymbol{x}) R_{i} \psi_{a}(\boldsymbol{x}) \theta_{c}(\boldsymbol{x}) \hat{p}_{k}(\tilde{\boldsymbol{x}}) d S_{x} d S_{\tilde{x}} \\
& -\rho \omega^{2} \int_{S_{p}} \int_{\Gamma} U_{a}^{k}(\tilde{\boldsymbol{x}}, \boldsymbol{x}) \psi_{a}(\boldsymbol{x}) n_{c}(\boldsymbol{x}) \theta_{c}(\boldsymbol{x}) \hat{p}_{k}(\tilde{\boldsymbol{x}}) d S_{x} d S_{\tilde{x}}
\end{aligned}
$$


Equations (55) and (56) are direct consequences of the symmetry properties $\mathcal{B}_{p \phi}(\boldsymbol{p}, \boldsymbol{\psi})=\mathcal{B}_{\phi p}(\boldsymbol{\psi}, \boldsymbol{p})$ and $\mathcal{B}_{u \phi}(\boldsymbol{u}, \boldsymbol{\psi})=\mathcal{B}_{\phi u}^{\star}(\boldsymbol{\psi}, \boldsymbol{u})$, respectively. The new kernels $B_{i k q s}^{\star}$ and $A_{i k}^{\star}$ in Eq. (57) are:

$$
\begin{gathered}
B_{i k q s}^{\star}(\tilde{\boldsymbol{x}}, \boldsymbol{x} ; \boldsymbol{\theta})=D_{q} \theta_{c}(\tilde{\boldsymbol{x}}) B_{i k c s}(\tilde{\boldsymbol{x}}, \boldsymbol{x})+D_{s} \theta_{c}(\boldsymbol{x}) B_{i k q c}(\tilde{\boldsymbol{x}}, \boldsymbol{x})+\left[\theta_{c}(\boldsymbol{x})-\theta_{c}(\tilde{\boldsymbol{x}})\right] B_{i k q s, c}(\tilde{\boldsymbol{x}}, \boldsymbol{x}) \\
A_{i k}^{\star}(\tilde{\boldsymbol{x}}, \boldsymbol{x} ; \boldsymbol{\theta})=\mu^{2}\left\{A_{i j k \ell, c}(\tilde{\boldsymbol{x}}, \boldsymbol{x})\left[\theta_{c}(\boldsymbol{x})-\theta_{c}(\tilde{\boldsymbol{x}})\right] n_{j}(\tilde{\boldsymbol{x}}) n_{\ell}(\boldsymbol{x})\right. \\
\left.+\left[e_{\ell c d} R_{d} \theta_{c}(\boldsymbol{x}) n_{j}(\tilde{\boldsymbol{x}})+e_{j c d} R_{d} \theta_{c}(\tilde{\boldsymbol{x}}) n_{\ell}(\boldsymbol{x})\right] A_{i j k \ell}(\tilde{\boldsymbol{x}}, \boldsymbol{x})\right\}
\end{gathered}
$$

where the coefficients $A_{i j k \ell}$ are defined by Eq. (90). The first two equations in (52) can in fact be obtained simply by multiplying Eq (33) (resp. Eq (34)) by a trial traction $\boldsymbol{q}(\tilde{\boldsymbol{x}})$ (resp. a trial displacement $\boldsymbol{v}(\tilde{\boldsymbol{x}})$ ) and integrating the result over $\tilde{\boldsymbol{x}} \in S_{u}$ (resp. $\tilde{\boldsymbol{x}} \in S_{p}$ ). The third equation in (52) is obtained by taking the material derivative of the third equation of the initial SGBIE system (38). In particular, to establish Eq. (57), use is made of:

$$
\left(R_{i} u \mathrm{~d} S\right)^{\star}=R_{a} u D_{a} \theta_{i}
$$

(see proof in Appendix A.5).

\section{Crack shape sensitivity of functionals using direct differentia- tion}

\subsection{Objective function}

Let us introduce the following generic objective function:

$$
\mathcal{J}(\Gamma)=J\left(\boldsymbol{u}_{\Gamma}, \boldsymbol{p}_{\Gamma}, \Gamma\right)=\int_{S_{p}} \varphi_{u}\left(\boldsymbol{u}_{\Gamma}, \boldsymbol{x}\right) \mathrm{d} S+\int_{S_{u}} \varphi_{p}\left(\boldsymbol{p}_{\Gamma}, \boldsymbol{x}\right) \mathrm{d} S+\int_{\Gamma} \psi(\boldsymbol{x}) \mathrm{d} S
$$

where $\varphi_{u}, \varphi_{p}$ and $\psi$ are real-valued differentiable functions and $\left(\boldsymbol{u}_{\Gamma}, \boldsymbol{p}_{\Gamma}\right)$ refer to the solution of problem $(1,2)$ for a given crack configuration. A notable example of such functions occurs in crack identification problems, where supplementary boundary data in the form of measured displacement $\boldsymbol{u}^{e x p}$ on $S_{p}$ and measured tractions $\boldsymbol{p}^{\text {exp }}$ on $S_{u}$ is available and one wants to find $\Gamma$ by minimizing the cost function:

$$
\mathcal{J}(\Gamma)=\frac{1}{2} \int_{S_{p}}\left|\boldsymbol{u}_{\Gamma}-\boldsymbol{u}^{e x p}\right|^{2} \mathrm{~d} S+\frac{1}{2} \int_{S_{u}}\left|\boldsymbol{p}_{\Gamma}-\boldsymbol{p}^{e x p}\right|^{2} \mathrm{~d} S+\alpha \int_{\Gamma} \psi(\boldsymbol{x}) \mathrm{d} S
$$

(i.e. corresponding to the choice $\varphi_{u} \equiv\left|\boldsymbol{u}-\boldsymbol{u}^{\exp }\right|^{2} / 2$ and $\varphi_{p} \equiv\left|\boldsymbol{p}-\boldsymbol{p}^{\exp }\right|^{2} / 2$ in Eq. (63)) where the last integral is chosen so as to express some a priori information of a geometrical nature about the unknown crack, for instance by penalizing highly oscillatory shapes, and $0<\alpha \ll 1$ is a regularization parameter $[28,44]$.

The remainder of this paper is devoted to various BEM-oriented procedures allowing to evaluate the derivative of $\mathcal{J}(\Gamma)$ with respect to crack perturbations. 


\subsection{Direct differentiation approach}

A direct application of formula (14) to (63) yields, taking into account that $\boldsymbol{\theta}=\boldsymbol{\nabla} \boldsymbol{\theta}=\mathbf{0}$ on $S_{u}$ and $S_{p}$ :

$$
\stackrel{\star}{\mathcal{J}}(\Gamma)=\int_{S_{p}} \frac{\partial \bar{\varphi}_{u}}{\partial \boldsymbol{u}} \cdot \stackrel{\star}{\boldsymbol{u}} \mathrm{d} S+\int_{S_{u}} \frac{\partial \bar{\varphi}_{p}}{\partial \boldsymbol{p}} \cdot \stackrel{\star}{\boldsymbol{p}} \mathrm{d} S+\int_{\Gamma}\left[\boldsymbol{\nabla} \psi \cdot \boldsymbol{\theta}+\psi \operatorname{div}_{S} \boldsymbol{\theta}\right] \mathrm{d} S
$$

The direct differentiation approach consists in solving the derivative BEM systems, in either the collocation form (37) or the Galerkin form (52), for the material derivatives $\stackrel{\star}{\boldsymbol{u}}, \stackrel{\star}{\boldsymbol{p}}, \stackrel{\star}{\phi}$. In practice, the crack shape will be defined in terms of a finite number $N_{p}$ of shape parameters $t_{m}, 1 \leq m \leq N_{p}$, so that the geometrical transformation (3). Each shape parameter must then be associated to a transformation velocity field $\boldsymbol{\theta}^{m}(\boldsymbol{x})$ through the definition (4). Thus, this process leads to the construction of $N_{p}$ distinct right-hand sides for the derivative BEM systems (37) or (52), and therefore $N_{p}$ distinct derivative solutions $\left(\stackrel{\star}{\boldsymbol{p}} m, \stackrel{\star}{\boldsymbol{u}}^{m}, \stackrel{\star}{\phi}^{m}\right)$. Finally, substituting the derivative solution $\left(\stackrel{\boldsymbol{p}}{m}^{m}, \stackrel{\star}{\boldsymbol{u}}^{m}, \stackrel{\star}{\phi}^{m}\right)$ into Eq. (65) yields the partial derivative of $\mathcal{J}$ with respect to the parameter $t_{m}$.

\section{Crack shape sensitivity of functionals using an adjoint solution}

As pointed out previously, the direct differentiation approach calls for building and solving $N_{p}$ derivative problems, i.e. one for each geometrical parameter; this is true independently of the number $N_{f}$ of integral functionals of the form (63) considered (one often has $N_{f}$ but this is not mandatory).

On the other hand, it is possible to replace the computation of the $N_{p}$ derivative solutions

$\left(\stackrel{\star}{\boldsymbol{p}}^{m}, \stackrel{\star}{\boldsymbol{u}}^{m}, \stackrel{\star}{\phi}^{m}\right)$ by that of $N_{f}$ well-chosen adjoint solutions, i.e. one for each integral functional (63). The basic argument, which has been used in many other works [1, 4, 17-20,34], goes as follows: to evaluate the derivative of $\mathcal{J}(\Gamma)$, the differentiation of $J(\boldsymbol{u}, \boldsymbol{p}, \Gamma)$ is carried out by formally treating $(\boldsymbol{u}, \boldsymbol{p}, \Gamma)$ as independent variables and enforcing the fact that $(\boldsymbol{u}, \boldsymbol{p})$ depend on $\Gamma$ through the state equations in an explicit manner. A Lagrangian is thus introduced, where the state equations appear as a constraint.

Three different approaches to the definition of adjoint solutions, all of them well suited to crack shape sensitivity analysis in a BEM framework, are now going to be presented.

\subsection{State equation in collocation BIE form}

In this case, the state equation consists of the system (32), in matrix form, i.e. after a BEM discretization has been introduced. The Lagrangian $\mathcal{L}$ is thus defined as follows, in terms of finite- 
dimensional vectors of Lagrange multipliers $\boldsymbol{q}$ and $\boldsymbol{\psi}$ :

$$
\mathcal{L}=J(\boldsymbol{u}, \boldsymbol{p}, \Gamma)+\left\{\begin{array}{c}
\boldsymbol{q} \\
\boldsymbol{\psi}
\end{array}\right\}^{T}\left\{\begin{array}{l}
\mathcal{H}_{p}(\boldsymbol{p})+\mathcal{G}_{u}(\boldsymbol{u})+\mathcal{G}_{\Gamma}(\phi)+\mathcal{H}_{u}(\hat{\boldsymbol{p}})+\mathcal{G}_{p}(\hat{\boldsymbol{u}}) \\
\mathcal{S}_{p}(\boldsymbol{p})+\mathcal{D}_{u}(\boldsymbol{u})+\mathcal{D}_{\Gamma}(\boldsymbol{\phi})+\mathcal{S}_{p}(\hat{\boldsymbol{p}})+\mathcal{D}_{u}(\hat{\boldsymbol{u}})
\end{array}\right\}
$$

$(\boldsymbol{q}, \boldsymbol{\psi}$ are thus 'vectors' of DOFs associated with the collocation points on $S$ and $\Gamma$, respectively). Putting the material derivative of $J$, Eq. (65), in the form

$$
\stackrel{\star}{\mathcal{J}}=\boldsymbol{J}_{p}(\boldsymbol{p}) \stackrel{\star}{\boldsymbol{p}}+\boldsymbol{J}_{u}(\boldsymbol{u}) \stackrel{\star}{\boldsymbol{u}}+\boldsymbol{J}_{\Gamma} \boldsymbol{\theta}
$$

and taking the material derivative of $\mathcal{L}$ gives:

$$
\begin{aligned}
\stackrel{\star}{\mathcal{L}}= & \boldsymbol{J}_{p}(\boldsymbol{p}) \stackrel{\star}{\boldsymbol{p}}+\boldsymbol{J}_{u}(\boldsymbol{u}) \stackrel{\star}{\boldsymbol{u}}+\boldsymbol{J}_{\Gamma} \boldsymbol{\theta}+\left\{\begin{array}{l}
\boldsymbol{q} \\
\boldsymbol{\psi}
\end{array}\right\}^{T}\left\{\begin{array}{l}
\mathcal{H}_{p}(\stackrel{\star}{\boldsymbol{p}})+\mathcal{G}_{u}(\stackrel{\star}{\boldsymbol{u}})+\mathcal{G}_{\phi}(\stackrel{\star}{\boldsymbol{\phi}}) \\
\mathcal{S}_{p}(\stackrel{\star}{\boldsymbol{p}})+\mathcal{D}_{u}\left({ }^{\star}\right)+\mathcal{D}_{\phi}(\stackrel{\star}{\boldsymbol{\phi}})
\end{array}\right\} \\
& +\left\{\begin{array}{c}
\boldsymbol{q} \\
\boldsymbol{\psi}
\end{array}\right\}^{T}\left\{\begin{array}{l}
\mathcal{G}_{\phi}^{\star}(\boldsymbol{\phi} ; \boldsymbol{\theta}) \\
\mathcal{S}_{p}^{\star}(\boldsymbol{p} ; \boldsymbol{\theta})+\mathcal{D}_{u}^{\star}(\boldsymbol{u} ; \boldsymbol{\theta})+\mathcal{D}_{\phi}^{\star}(\boldsymbol{\phi} ; \boldsymbol{\theta})+\mathcal{S}_{u}^{\star}(\hat{\boldsymbol{p}} ; \boldsymbol{\theta})+\mathcal{D}_{p}^{\star}(\hat{\boldsymbol{u}} ; \boldsymbol{\theta})+\mathcal{S}_{u}(\stackrel{\star}{\hat{\boldsymbol{p}}})+\mathcal{D}_{p}\left({ }^{\star} \boldsymbol{\boldsymbol { u }}\right)
\end{array}\right\}
\end{aligned}
$$

As expected, this equation involves the derivatives $\stackrel{\star}{\boldsymbol{p}}, \stackrel{\star}{\boldsymbol{u}}, \stackrel{\star}{\boldsymbol{\phi}}$ of the state variables. It is however possible to select a particular value of the Lagrange multipliers $\{\boldsymbol{q}, \boldsymbol{\psi}\}^{T}$ which makes the overall contribution of $\stackrel{\star}{\boldsymbol{p}}, \stackrel{\star}{\boldsymbol{u}}, \stackrel{\star}{\phi}$ in $\stackrel{\star}{\mathcal{L}}$ identically zero. This specific value of the multipliers, termed an adjoint solution, thus results from the condition:

$$
\boldsymbol{J}_{p}(\boldsymbol{p}) \stackrel{\star}{\boldsymbol{p}}+\boldsymbol{J}_{u}(\boldsymbol{u}) \stackrel{\star}{\boldsymbol{u}}+\left\{\begin{array}{c}
\boldsymbol{q} \\
\boldsymbol{\psi}
\end{array}\right\}^{T}\left\{\begin{array}{l}
\mathcal{H}_{p}(\stackrel{\star}{\boldsymbol{p}})+\mathcal{G}_{u}(\stackrel{\star}{\boldsymbol{u}})+\mathcal{G}_{\phi}(\stackrel{\star}{\boldsymbol{\phi}}) \\
\mathcal{S}_{p}\left({ }^{\star}\right)+\mathcal{D}_{u}\left(\stackrel{\star}{\boldsymbol{u}}^{\boldsymbol{p}}\right)+\mathcal{D}_{\phi}(\stackrel{\star}{\boldsymbol{\phi}})
\end{array}\right\}=0 \quad \text { for all } \stackrel{\star}{\boldsymbol{p}}, \stackrel{\star}{\boldsymbol{u}}, \stackrel{\star}{\boldsymbol{\phi}}
$$

and solves the adjoint problem:

$$
\left\{\begin{array}{l}
\mathcal{H}_{p}^{T}(\stackrel{\star}{\boldsymbol{p}})+\mathcal{S}_{p}^{T}(\stackrel{\star}{\boldsymbol{p}})=-\boldsymbol{J}_{p}(\boldsymbol{p}) \\
\mathcal{G}_{u}^{T}(\stackrel{\star}{\boldsymbol{u}})+\mathcal{D}_{u}^{T}(\stackrel{\star}{\boldsymbol{u}})=-\boldsymbol{J}_{u}(\boldsymbol{u}) \\
\mathcal{G}_{\phi}^{T}(\stackrel{\star}{\boldsymbol{\phi}})+\mathcal{D}_{\phi}^{T}(\stackrel{\star}{\boldsymbol{\phi}})=0
\end{array}\right.
$$

It is important to note that the governing matrix of the adjoint problem is the transpose of that of the state equations (32). The factorization (using e.g. the LU decomposition) made in the course of solving the state BIE equations can thus be re-used to solve the adjoint problem (70).

Using the solution $\left\{\boldsymbol{q}_{\Gamma}, \boldsymbol{\psi}_{\Gamma}\right\}^{T}$ of the adjoint problem (70), the material derivative of $J$ in any given crack perturbation can thus be computed by the formula:

$$
\begin{aligned}
& \stackrel{\star}{J}=\boldsymbol{J}_{\Gamma} \boldsymbol{\theta}+\boldsymbol{q}_{\Gamma}^{T} \mathcal{G}_{\phi}^{\star}\left(\phi_{\Gamma} ; \boldsymbol{\theta}\right) \\
& +\boldsymbol{\psi}_{\Gamma}\left[\mathcal{G}_{\phi}^{\star}\left(\phi_{\Gamma} ; \boldsymbol{\theta}\right) \mathcal{S}_{p}^{\star}\left(\boldsymbol{p}_{\Gamma} ; \boldsymbol{\theta}\right)+\mathcal{D}_{u}^{\star}\left(\boldsymbol{u}_{\Gamma} ; \boldsymbol{\theta}\right)+\mathcal{D}_{\phi}^{\star}\left(\phi_{\Gamma} ; \boldsymbol{\theta}\right)+\mathcal{S}_{u}^{\star}(\hat{\boldsymbol{p}} ; \boldsymbol{\theta})+\mathcal{D}_{p}^{\star}(\hat{\boldsymbol{u}} ; \boldsymbol{\theta})+\mathcal{S}_{u}(\hat{\boldsymbol{p}})+\mathcal{D}_{p}(\hat{\boldsymbol{u}})\right]
\end{aligned}
$$

where $\left(\boldsymbol{p}_{\Gamma}, \boldsymbol{u}_{\Gamma}, \phi_{\Gamma}\right)$ and $\left(\boldsymbol{q}_{\Gamma}, \boldsymbol{\psi}_{\Gamma}\right)$ denote the direct and adjoint solutions for the current crack configuration $\Gamma$. 


\subsection{State equation in Galerkin BIE form}

It is also possible to define an adjoint problem from the continuous state equations, i.e. before their discretization, and many examples of that can be found in the literature. For example, one can think of taking the inner product of the collocation BIE equation (29) and (31) by continuous Lagrange multipliers $\boldsymbol{q}(\tilde{\boldsymbol{x}})$ and $\boldsymbol{\psi}(\tilde{\boldsymbol{x}})$ and integrate the result over $S$ and $S_{p}$ respectively. But this operation amounts to forming a set of (nonsymmetric) Galerkin BIEs. Similarly, taking the inner product of equations (29), (30) and (31) by Lagrange multipliers $\boldsymbol{q}(\tilde{\boldsymbol{x}}), \boldsymbol{v}(\tilde{\boldsymbol{x}})$ and $\boldsymbol{\psi}(\tilde{\boldsymbol{x}})$ and integrating the resulting expressions over $S_{u}, S_{p}$ and $\Gamma$, respectively, yields the system (38) of symmetric Galerkin BIEs.

Following the same steps than before, one can then introduce a Lagrangian in which the direct problem constraint is expressed in terms of the symmetric Galerkin BIE system (38), the Lagrange multipliers being the test functions $(\boldsymbol{v}, \boldsymbol{q}, \boldsymbol{\psi})$ :

$$
\begin{aligned}
& \mathcal{L}(\boldsymbol{u}, \boldsymbol{v}, \boldsymbol{p}, \boldsymbol{q}, \boldsymbol{\phi}, \boldsymbol{\psi}, \Gamma)=J(\boldsymbol{u}, \boldsymbol{p}, \Gamma) \\
& \quad+\mathcal{B}_{p p}(\boldsymbol{p}, \boldsymbol{q})+\mathcal{B}_{u p}(\boldsymbol{u}, \boldsymbol{q})+\mathcal{B}_{\phi p}(\boldsymbol{\phi}, \boldsymbol{q})-\mathcal{F}_{u p}(\hat{\boldsymbol{u}}, \boldsymbol{q})-\mathcal{F}_{p p}(\hat{\boldsymbol{p}}, \boldsymbol{q}) \\
& \quad+\mathcal{B}_{p u}(\boldsymbol{p}, \boldsymbol{v})+\mathcal{B}_{u u}(\boldsymbol{u}, \boldsymbol{v})+\mathcal{B}_{\phi u}(\boldsymbol{\phi}, \boldsymbol{v})-\mathcal{F}_{u u}(\hat{\boldsymbol{u}}, \boldsymbol{v})-\mathcal{F}_{u p}(\hat{\boldsymbol{p}}, \boldsymbol{v}) \\
& \quad+\mathcal{B}_{p \phi}(\boldsymbol{p}, \boldsymbol{\psi})+\mathcal{B}_{u \phi}(\boldsymbol{u}, \boldsymbol{\psi})+\mathcal{B}_{\phi \phi}(\boldsymbol{\phi}, \boldsymbol{\psi})-\mathcal{F}_{u \phi}(\hat{\boldsymbol{u}}, \boldsymbol{\psi})-\mathcal{F}_{u \phi}(\hat{\boldsymbol{p}}, \boldsymbol{\psi})
\end{aligned}
$$

The derivative $\stackrel{\star}{\mathcal{L}}$ is then expressed using Eq. (67) and the system (52) of derivative Galerkin BIEs, and the adjoint problem, again defined to ensure that the contribution of $(\stackrel{\star \boldsymbol{p}}{\stackrel{\star}{\boldsymbol{u}}, \stackrel{\star}{\boldsymbol{\phi}})}$ is identically zero, is readily found to be defined by:

$$
\left\{\begin{array}{l}
\mathcal{B}_{p p}(\boldsymbol{q}, \stackrel{\star}{\boldsymbol{p}})+\mathcal{B}_{u p}(\boldsymbol{v}, \stackrel{\star}{\boldsymbol{p}})+\mathcal{B}_{\phi p}(\boldsymbol{\psi}, \stackrel{\star}{\boldsymbol{p}})=-\boldsymbol{J}_{p}(\boldsymbol{p}) \stackrel{\star}{\boldsymbol{p}} \\
\mathcal{B}_{p u}(\boldsymbol{q}, \stackrel{\star}{\boldsymbol{u}})+\mathcal{B}_{u u}(\boldsymbol{v}, \stackrel{\star}{\boldsymbol{u}})+\mathcal{B}_{\phi u}(\boldsymbol{\psi}, \stackrel{\star}{\boldsymbol{u}})=-\boldsymbol{J}_{u}(\boldsymbol{u}) \stackrel{\star}{\boldsymbol{u}} \\
\mathcal{B}_{p \phi}(\boldsymbol{q}, \stackrel{\star}{\boldsymbol{\phi}})+\mathcal{B}_{u \phi}(\boldsymbol{v}, \stackrel{\star}{\boldsymbol{\phi}})+\mathcal{B}_{\phi \phi}(\boldsymbol{\psi}, \stackrel{\star}{\boldsymbol{\phi}})=0
\end{array} \quad \text { for all } \stackrel{\star}{\boldsymbol{p}}, \stackrel{\star}{\boldsymbol{u}}, \stackrel{\star}{\boldsymbol{\phi}}\right.
$$

The material derivative of $J$ in any given crack perturbation is then given by:

$$
\begin{aligned}
\stackrel{\star}{J}=\stackrel{\star}{\mathcal{L}}\left(\boldsymbol{u}_{\Gamma}, \boldsymbol{v}_{\Gamma}, \boldsymbol{p}_{\Gamma}, \boldsymbol{q}_{\Gamma}, \boldsymbol{\phi}_{\Gamma}, \boldsymbol{\psi}_{\Gamma}, \boldsymbol{\theta}\right) \\
=\boldsymbol{J}_{\Gamma} \boldsymbol{\theta}+\mathcal{B}_{\phi p}^{\star}\left(\phi_{\Gamma}, \boldsymbol{q}_{\Gamma} ; \boldsymbol{\theta}\right)+\mathcal{B}_{\phi u}^{\star}\left(\phi_{\Gamma}, \boldsymbol{v}_{\Gamma} ; \boldsymbol{\theta}\right)+\mathcal{B}_{p \phi}^{\star}\left(\boldsymbol{p}_{\Gamma}, \boldsymbol{\psi}_{\Gamma} ; \boldsymbol{\theta}\right) \\
\quad+\mathcal{B}_{u \phi}^{\star}\left(\boldsymbol{u}_{\Gamma}, \boldsymbol{\psi}_{\Gamma} ; \boldsymbol{\theta}\right)+\mathcal{B}_{\phi \phi}^{\star}\left(\phi_{\Gamma}, \boldsymbol{\psi}_{\Gamma} ; \boldsymbol{\theta}\right)+\mathcal{F}_{u \phi}^{\star}\left(\hat{\boldsymbol{u}}_{\Gamma}, \boldsymbol{\psi}_{\Gamma} ; \boldsymbol{\theta}\right)+\mathcal{F}_{p \phi}^{\star}\left(\hat{\boldsymbol{p}}_{\Gamma}, \boldsymbol{\psi}_{\Gamma} ; \boldsymbol{\theta}\right) \\
\quad+\mathcal{F}_{u u}\left(\stackrel{\star}{\hat{\boldsymbol{u}}}, \boldsymbol{v}_{\Gamma}\right)+\mathcal{F}_{p u}\left(\stackrel{\star}{\hat{\boldsymbol{p}}}, \boldsymbol{v}_{\Gamma}\right)+\mathcal{F}_{u p}\left(\stackrel{\star}{\hat{\boldsymbol{u}}}, \boldsymbol{q}_{\Gamma}\right)+\mathcal{F}_{p p}\left(\stackrel{\star}{\hat{\boldsymbol{p}}}, \boldsymbol{q}_{\Gamma}\right)+\mathcal{F}_{u \phi}(\stackrel{\star}{\hat{\boldsymbol{u}}}, \boldsymbol{q})+\mathcal{F}_{p \phi}(\stackrel{\hat{\boldsymbol{p}}}{, \boldsymbol{q}})
\end{aligned}
$$

\subsection{State equations are in domain form}

This is usually the simplest way to formulate an adjoint problem associated with a given integral functional, because the latter is then expressed as the weak (domain) form of a boundary-value 
problem which can then be solved using any method. However, this approach is not straightforward when dealing with crack problems, for reasons related to the fact that the direct and adjoint solutions have singular strains and stresses near the crack front. An appropriate answer to this difficulty has been proposed in [8] for time-domain elastodynamics, the results of which are now summarized for the sake of completeness.

The Lagrangian incorporates the state problem constraint in its usual domain weak form:

$$
\begin{aligned}
\mathcal{L}(\boldsymbol{u}, \boldsymbol{v}, \boldsymbol{p}, \boldsymbol{q}, \Gamma)=J(\boldsymbol{u}, \boldsymbol{p}, \Gamma)+\int_{\Omega}[\boldsymbol{\sigma}(\boldsymbol{u}): \nabla \overline{\boldsymbol{v}} & \left.-\rho \omega^{2} \boldsymbol{u} \cdot \overline{\boldsymbol{v}}\right] \mathrm{d} V \\
& -\int_{S_{u}}(\boldsymbol{u}-\hat{\boldsymbol{u}}) \cdot \overline{\boldsymbol{q}} \mathrm{d} S-\int_{S_{u}} \boldsymbol{p} \cdot \overline{\boldsymbol{v}} \mathrm{d} S-\int_{S_{p}} \hat{\boldsymbol{p}} \cdot \overline{\boldsymbol{v}} \mathrm{d} S
\end{aligned}
$$

Next, the derivative of the Lagrangian is found to be given by:

$$
\begin{aligned}
\stackrel{\star}{\mathcal{L}}(\boldsymbol{u}, \boldsymbol{v}, \boldsymbol{p}, \boldsymbol{q}, \boldsymbol{\theta})= & \int_{\Omega}\left[\boldsymbol{\sigma}(\overline{\boldsymbol{v}}): \nabla \stackrel{\star}{\boldsymbol{u}}-\rho \omega^{2} \overline{\boldsymbol{v}} \cdot \stackrel{\star}{\boldsymbol{u}}\right] \mathrm{d} V+\int_{S_{u}} \stackrel{\star}{\hat{\boldsymbol{u}}} \cdot \overline{\boldsymbol{q}} \mathrm{d} S-\int_{S_{p}} \stackrel{\star}{\hat{\boldsymbol{p}}} \cdot \overline{\boldsymbol{v}} \mathrm{d} S \\
& -\int_{S_{u}}\left(\overline{\boldsymbol{v}}-\frac{\partial \bar{\varphi}_{p}}{\partial \boldsymbol{p}}\right) \cdot \stackrel{\star}{\boldsymbol{p}} \mathrm{d} S-\int_{S_{u}} \overline{\boldsymbol{q}} \cdot \stackrel{\star}{\boldsymbol{u}} \mathrm{d} S+\int_{S_{p}} \frac{\partial \bar{\varphi}_{u}}{\partial \boldsymbol{u}} \cdot \stackrel{\star}{\boldsymbol{u}} \mathrm{d} S \\
& +\int_{\Omega}\left\{\left[\boldsymbol{\sigma}(\boldsymbol{u}): \nabla \overline{\boldsymbol{v}}-\rho \omega^{2} \boldsymbol{u} \cdot \overline{\boldsymbol{v}}\right] \operatorname{div} \boldsymbol{\theta}-[\boldsymbol{\sigma}(\boldsymbol{u}) \cdot \nabla \overline{\boldsymbol{v}}+\boldsymbol{\sigma}(\overline{\boldsymbol{v}}) \cdot \nabla \boldsymbol{u}]: \nabla \boldsymbol{\theta}\right\} \mathrm{d} V \\
& +\int_{\Gamma}\left[\nabla \psi \cdot \boldsymbol{\theta}+\psi \operatorname{div}_{S} \boldsymbol{\theta}\right] \mathrm{d} S
\end{aligned}
$$

so that, following the now usual argument, the adjoint solution $(\boldsymbol{v}, \boldsymbol{q})$ is such that all terms involving $(\stackrel{\star}{\boldsymbol{u}}, \stackrel{\star}{\boldsymbol{p}})$ in the formula above identically cancel out, and hence is found to be governed by the field equations (1) and the boundary conditions:

$$
\begin{gathered}
\boldsymbol{q}=-\frac{\partial \varphi_{u}}{\partial \boldsymbol{u}} \quad\left(\text { on } S_{p}\right) \\
\boldsymbol{v}=\frac{\partial \varphi_{p}}{\partial \boldsymbol{p}}\left(\text { on } S_{u}\right) \\
\boldsymbol{q}=\mathbf{0}\left(\text { on } \Gamma^{ \pm}\right)
\end{gathered}
$$

Then, the sensitivity $\stackrel{\star}{J}$ is obtained as usual by:

$$
\begin{aligned}
& \stackrel{\star}{\mathcal{J}}(\Gamma)=\stackrel{\star}{\mathcal{L}}\left(\boldsymbol{u}_{\Gamma}, \boldsymbol{v}_{\Gamma}, \boldsymbol{p}_{\Gamma}, \boldsymbol{q}_{\Gamma}, \Gamma\right) \\
& =\int_{\Omega}\left\{\left[\boldsymbol{\sigma}\left(\boldsymbol{u}_{\Gamma}\right): \nabla \overline{\boldsymbol{v}}_{\Gamma}-\rho \omega^{2} \boldsymbol{u} \cdot \overline{\boldsymbol{v}}\right] \operatorname{div} \boldsymbol{\theta}-\left[\boldsymbol{\sigma}\left(\boldsymbol{u}_{\Gamma}\right) \cdot \boldsymbol{\nabla} \overline{\boldsymbol{v}}_{\Gamma}+\boldsymbol{\sigma}\left(\overline{\boldsymbol{v}}_{\Gamma}\right) \cdot \boldsymbol{\nabla} \boldsymbol{u}_{\Gamma}\right]: \boldsymbol{\nabla} \boldsymbol{\theta}\right\} \mathrm{d} V \\
& +\int_{\Gamma}\left[\nabla \psi \cdot \boldsymbol{\theta}+\psi \operatorname{div}_{S} \boldsymbol{\theta}\right] \mathrm{d} S+\int_{S_{u}} \stackrel{\star}{\hat{\boldsymbol{u}}} \cdot \overline{\boldsymbol{q}} \mathrm{d} S-\int_{S_{p}} \stackrel{\star}{\hat{\boldsymbol{p}}} \cdot \overline{\boldsymbol{v}} \mathrm{d} S
\end{aligned}
$$

which is unfortunately not in boundary-only form, and thus unsuitable for boundary element methods. A boundary-only sensitivity formula can be obtained by an application of the divergence formula thanks to the following identity, valid for any pair $(\boldsymbol{u}, \boldsymbol{v})$ of solutions of the field equations 
(1):

$$
\begin{aligned}
{\left[\boldsymbol{\sigma}(\boldsymbol{u}): \nabla \overline{\boldsymbol{v}}-\rho \omega^{2} \boldsymbol{u} \cdot \overline{\boldsymbol{v}}\right] \operatorname{div} \boldsymbol{\theta}-[\boldsymbol{\sigma}(\boldsymbol{u}) \cdot \boldsymbol{\nabla} \overline{\boldsymbol{v}}+\boldsymbol{\sigma}(\overline{\boldsymbol{v}}) \cdot \boldsymbol{\nabla} \boldsymbol{u}]: \boldsymbol{\nabla} \boldsymbol{\theta} } \\
\quad=\operatorname{div}\left(\left[\boldsymbol{\sigma}(\boldsymbol{u}): \nabla \overline{\boldsymbol{v}}-\rho \omega^{2} \boldsymbol{u} \cdot \overline{\boldsymbol{v}}\right] \boldsymbol{\theta}-[\boldsymbol{\sigma}(\boldsymbol{u}) \cdot \boldsymbol{\nabla} \overline{\boldsymbol{v}}+\boldsymbol{\sigma}(\overline{\boldsymbol{v}}) \cdot \boldsymbol{\nabla u}] \cdot \boldsymbol{\theta}\right)
\end{aligned}
$$

For smooth-shaped defects like voids, this manoeuvre is sufficient. Here, however, the singular nature of the various strain and stress fields near the crack front calls for a careful treatment whereby a tubular neighbourhood of the crack front, with small radius $\varepsilon$, is introduced. The domain integral over this tubular neighbourhood, in the limit $\varepsilon \rightarrow 0$, is found to be entirely determined by the distributions on $\partial \Gamma$ of the stress intensity factors $\left(K_{j}^{u}(s), K_{j}^{v}(s) ; j=I, I I, I I I\right)$ of the direct and adjoint solutions $(s$ : arc length along $\partial \Gamma)$. The final, boundary-only, sensitivity formula for crack perturbations is:

$$
\begin{aligned}
\stackrel{\star}{\mathcal{J}}(\Gamma)= & \int_{\Gamma}(\boldsymbol{\theta} \cdot \boldsymbol{n})(s) \llbracket \boldsymbol{\sigma}(\boldsymbol{u}): \nabla \overline{\boldsymbol{v}}-\rho \omega^{2} \boldsymbol{u} \cdot \overline{\boldsymbol{v}} \rrbracket \mathrm{d} S+\int_{S_{u}} \hat{\boldsymbol{u}} \cdot \overline{\boldsymbol{q}} \mathrm{d} S-\int_{S_{p}} \hat{\boldsymbol{p}} \cdot \overline{\boldsymbol{v}} \mathrm{d} S \\
& -\frac{1}{\mu} \int_{\partial \Gamma}(\boldsymbol{\theta} \cdot \boldsymbol{\nu})(s)\left\{(1-\nu)\left[K_{I}^{u} \bar{K}_{I}^{v}+K_{I I}^{u} \bar{K}_{I I}^{v}\right]+K_{I I I}^{u} \bar{K}_{I I I}^{v}\right\}(s) \mathrm{d} s \\
& +\frac{1-\nu}{\mu} \int_{\partial \Gamma}(\boldsymbol{\theta} \cdot \boldsymbol{n})(s)\left(K_{I}^{u} \bar{K}_{I I}^{v}+K_{I I}^{u} \bar{K}_{I}^{v}\right)(s) \mathrm{d} s
\end{aligned}
$$

where $\llbracket f \rrbracket \equiv f^{+}-f^{-}$denotes the discontinuity of $f$ across $\Gamma$. To ensure that formula (80) can actually be evaluated using only the boundary traces of the direct and adjoint solutions, the bilinear form $\boldsymbol{\sigma}(\boldsymbol{u}): \boldsymbol{\nabla} \overline{\boldsymbol{v}}$ must be expressed in terms of $\boldsymbol{\nabla}_{S} \boldsymbol{u}, \nabla_{S} \overline{\boldsymbol{v}}$, taking $\boldsymbol{p}=\boldsymbol{q}=\mathbf{0}$ into account in the process:

$$
\boldsymbol{\sigma}(\boldsymbol{u}): \boldsymbol{\nabla} \overline{\boldsymbol{v}}=\mu\left\{\frac{2 \nu}{1-\nu} \operatorname{div}_{S} \boldsymbol{u} \operatorname{div}_{S} \overline{\boldsymbol{v}}+\frac{1}{2}\left(\boldsymbol{\nabla}_{S} \boldsymbol{u}+\nabla_{S}^{T} \boldsymbol{u}\right):\left(\nabla_{S} \overline{\boldsymbol{v}}+\nabla_{S}^{T} \overline{\boldsymbol{v}}\right)-\left(\boldsymbol{n} \cdot \nabla_{S} \boldsymbol{u}\right) \cdot\left(\boldsymbol{n} \cdot \boldsymbol{\nabla}_{S} \overline{\boldsymbol{v}}\right)\right\}
$$

Some preliminary numerical results for two-dimensional time-domain elastodynamics using a dual BEM formulation and the two-dimensional time-domain analog of Eq. (80) have been presented in $[11]$.

\section{Discussion}

- The derivative integral identities (21) and (28) are not new. Indeed, essentially the same identities (or their obvious transpositions to e.g. elastostatics or potential problems) can be found in many other references, either using CPV- and FP-singular integrals $[2,23,33]$ or in an equivalent regularized form [5,32]. They are presented here in some detail because, in addition to their being essential for the remainder of this paper, we believe that, in contrast with some of the previously mentioned references where the material derivative formula is 
applied without justification to CPV- and FP-singular integrals, identities (21) and (28) are established on a sound basis.

- To perform a shape sensitivity analysis using any one of the methods presented here, the material derivative $(\stackrel{\star}{\hat{\boldsymbol{p}}}, \hat{\boldsymbol{u}})$ of the prescribed boundary data should be known in advance. In many situations, one can simply assume that

$$
\stackrel{\star}{\hat{p}}=\mathbf{0} \quad \stackrel{\star}{\hat{u}}=\mathbf{0}
$$

and the corresponding terms in Eqs. (71), (74) and (80) disappear. This is in particular the case for crack identification inverse problems.

- The adjoint solution approach is usually the most efficient one when the direct problem is linear (like here), because one usually consider one cost function, or perhaps a small number of them in multi-objective optimization, whereas many geometrical parameters may be involved. On the other hand, the direct differentation approach, through the solution of the derivative BIEs (37) or (52), yields the pointwise shape sensitivities of the elastodynamic field variables as well. This information is needed in the successive linearization treatments of inverse problems proposed in e.g. [24,25], as well as for computing an approximation of the Hessian based upon the first-order derivatives of residuals in the Marquardt-Levenberg or GaussNewton methods for least squares.

- Reference [9] (which deals with the computation of energy release rates and the incremental extension of cracks) essentially uses the adjoint solution approach based on the symmetric Galerkin BIE formulation as presented in section 8.2 (the cost function being the potential energy of elastic solids at equilibrium).

- Equation (80) is a generalization of some previously known identities. For instance, in elastostatics, $\mathcal{J}(\Gamma)$ is the potential energy at equilibrium for the particular choice $\varphi_{u}=-(\hat{\boldsymbol{p}} . \boldsymbol{u}) / 2$, $\varphi_{p}=(\hat{\boldsymbol{u}} \cdot \boldsymbol{p}) / 2$ in Eq. (63). For this special case, the adjoint solution turns out to be $\boldsymbol{v}=(1 / 2) \boldsymbol{u}$, i.e. $K_{I}^{v}=K_{I} / 2$, etc. In equation (80), the factor of $(\boldsymbol{\theta} \cdot \boldsymbol{\nu})(s)$ turns out to be, as expected, minus the energy release rate $G(s)$, i.e. minus the $J_{1}$-integral, whereas the factor of $(\boldsymbol{\theta} . \boldsymbol{n})(s)$ is the 3 -D generalization of the $J_{2}$-integral $[13,14]$. Finally, with the choice $S_{p}=S, s_{u}=\emptyset$ and $\varphi_{p}=\hat{\boldsymbol{p}} \cdot \hat{\boldsymbol{u}}-\boldsymbol{u} \cdot \hat{\boldsymbol{p}}$, where $\hat{\boldsymbol{u}}, \hat{\boldsymbol{p}}$ are the boundary traces of a pre-selected auxiliary elastodynamic state with final homogeneous conditions, one finds that $\boldsymbol{v}=\hat{\boldsymbol{u}}$ and that the factor of $(\boldsymbol{\theta} . \boldsymbol{\nu})(s)$ in (80) is the 3-D generalization of the so-called $H$-integral [16]. 
- The numerical evaluation of the sensitivity formula (80) is not possible using the displacement discontinuity BIE formulation alone, because the combination $\llbracket \boldsymbol{\sigma}(\boldsymbol{u}): \boldsymbol{\nabla} \overline{\boldsymbol{v}}-\rho \omega^{2} \boldsymbol{u} \cdot \overline{\boldsymbol{v}} \rrbracket$ is not expressible solely in terms of the jumps $\llbracket \boldsymbol{u} \rrbracket=\phi$ and $\llbracket \boldsymbol{v} \rrbracket=\boldsymbol{\psi}$. Instead, one should either solve the direct and adjoint problems by means of the dual BIE method or evaluate (say) $\boldsymbol{u}^{+}$ and $\boldsymbol{v}^{+}$using a representation formula.

\section{References}

[1] Aithal R, Saigal S. Shape sensitivity analysis in thermal problems using BEM. Engng. Anal. with Bound. Elem. 1995. 15:115-120.

[2] Barone MR, Yang RJ. A boundary element approach for recovery of shape sensitivities in three-dimensional elastic solids. Comp. Meth. in Appl. Mech. Engng. 1989. 74:69-82.

[3] Bécache E, Nedelec JC, Nishimura N. Regularization in 3D for anisotropic elastodynamic crack and obstacle problems. J. Elast. 1993. 31:25-46.

[4] Bonnet M. BIE and material differentiation applied to the formulation of obstacle inverse problems. Engng. Anal. with Bound. Elem. 1995. 15:121-136.

[5] Bonnet M. Regularized BIE formulations for first- and second-order shape sensitivity of elastic fields. Computers and Structures 1995. 56:799-811. (Invited paper, special issue, S. Saigal, guest editor).

[6] Bonnet M. Regularized direct and indirect symmetric variational BIE formulations for threedimensional elasticity. Engng. Anal. with Bound. Elem. 1995. 15:93-102.

[7] Bonnet M. Differentiability of strongly singular and hypersingular boundary integral formulations with respect to boundary perturbations. Comp. Mech. 1997. 19:240-246.

[8] Bonnet M. A general boundary-only formula for crack shape sensitivity of integral functionals. C.R. Acad. Sci. Paris, série II 1999. 327:1215-1221.

[9] Bonnet M. Stability of crack fronts under Griffith criterion: a computational approach using integral equations and domain derivatives of potential energy. Comp. Meth. in Appl. Mech. Engng. 1999. 173:337-364.

[10] Bonnet M, Bui HD. Regularization of the displacement and traction BIE for 3D elastodynamics using indirect methods. In JH Kane, G Maier, N Tosaka, SN Atluri, editors, Advances in Boundary Element Techniques. Springer-Verlag, 1993 1-29. 
[11] Bonnet M, Burczyński T, Nowakowski M. A BEM- and adjoint variable-based approach to crack shape sensitivity analysis. In K Woodbury, editor, Inverse problems in engineering : theory and practice. ASME, 2000, 2000 169-176.

[12] Bonnet M, Maier G, Polizzotto C. On symmetric galerkin boundary element method. Appl. Mech. Rev. 1998. 51:669-704.

[13] Budiansky B, Rice JR. Conservation laws and energy release rates. ASME J. Appl. Mech. 1973. 40:201-203.

[14] Bui HD. Mécanique de la rupture fragile. Masson, 1978.

[15] Bui HD, Bonnet M, editors. Unknown or variable domains, inverse problems., Engng. Anal. with Bound. Elem., vol. 15(2) (special issue), 1995.

[16] Bui HD, Maigre H, Rittel D. A new approach to the experimental determination of the dynamic stress intensity factor. Int. J. Solids Struct. 1992. 29:2881-2895.

[17] Burczyński T. Application of BEM in sensitivity analysis and optimization. Comp. Mech. 1993. 13:29-44.

[18] Burczyński T, Fedelinski P. Boundary elements in shape design sensitivity analysis and optimal design of vibrating structures. Engng. Anal. with Bound. Elem. 1992. 9:195-201.

[19] Burczyński T, Kane JH, Balakrishna C. Shape design sensitivity analysis via material derivative - adjoint variable approach. Int. J. Num. Meth. in Eng. 1995. 38:2839-2866.

[20] Choi JO, Kwak BM. Boundary Integral Equation Method for Shape Optimization of Elastic Structures. Int. J. Num. Meth. in Eng. 1988. 26:1579-1595.

[21] Dems K, Mróz Z. On a class of conservation rules associated with sensitivity analysis in linear elasticity. Int. J. Solids Struct. 1986. 22:737-758.

[22] Eringen AC, Suhubi ES. Elastodynamics (vol II - linear theory). Academic Press, 1975.

[23] Erman Z, Fenner RT. Design sensitivity analysis of three-dimensional elastostatic solids using the boundary integral equation method. In CA Brebbia, editor, Boundary Element Method XVI. Comp. Mech. Publ., Southampton, 1994, 1994 575-582.

[24] Gallego R, Suarez J. Numerical solution of the variation boundary integral equation for inverse problems. Int. J. Num. Meth. in Eng. 2000. 49:501-518. 
[25] Gallego R, Suarez J. Solution of inverse problems by boundary integral equations without residual minimization. Int. J. Solids Struct. 2000. 40:5629-5652.

[26] Guiggiani M. Formulation and numerical treatment of boundary integral equations with hypersingular kernels. In V Sladek, J Sladek, editors, Singular Integrals in Boundary Element Methods. Comp. Mech. Publ., Southampton, 1998 85-124.

[27] Guiggiani M, Gigante A. A general algorithm for multidimensional Cauchy principal value integrals in the boundary element method. ASME J. Appl. Mech. 1990. 57:906-915.

[28] Hansen PC. Rank-deficient and discrete ill-posed problems. SIAM, Philadelphia, USA, 1998.

[29] Haug EJ, Choi KK, Komkov V. Design Sensitivity Analysis of Structural Systems. Academic Press, 1986.

[30] Kleiber Mea. Parameter Sensitivity in Nonlinear Mechanics: Theory and Finite Element Computations. J. Wiley and Sons, New York, 1997.

[31] Krishnasamy G, Rizzo FJ, Rudolphi TJ. Hypersingular boundary integral equations: their occurrence, interpretation, regularization and computation. In PK Banerjee, S Kobayashi, editors, Developments in Boundary Element Methods, volume 7: Advanced Dynamic Analysis. Elsevier, 1992 207-252.

[32] Matsumoto T, Tanaka M, Miyagawa M, Ishii N. Optimum design of cooling lines in injection moulds by using boundary element design sensitivity analysis. Finite Elements in Analysis and Design 1993. 14:177-185.

[33] Mellings SC, Aliabadi MH. Flaw identification using the boundary element method. Int. J. Num. Meth. in Eng. 1995. 38:399-419.

[34] Meric RA. Differential and integral sensitivity formulations and shape optimization by BEM. Engng. Anal. with Bound. Elem. 1995. 15:181-188.

[35] Nedelec JC. Integral equations with non integrable kernels. Integral equations and operator theory 1982. 5:562-572.

[36] Nishimura N. Application of boundary integral equation method to various crack determination problems. In M Aliabadi, editor, Dynamic fracture mechanics. Comp. Mech. Publ., Southampton, 1995 . 
[37] Nishimura N, Furukawa A, Kobayashi S. Regularized boundary integral equations for an inverse problem of crack determination in time domain. In S Kobayashi, N Nishimura, editors, Boundary element methods. Springer-Verlag, 1992 252-261.

[38] Nishimura N, Kobayashi S. A regularized boundary integral equation method for elastodynamic crack problems. Comp. Mech. 1989. 4:319-328.

[39] Nishimura N, Kobayashi S. A boundary integral equation method for an inverse problem related to crack detection. Int. J. Num. Meth. in Eng. 1991. 32:1371-1387.

[40] Petryk H, Mróz Z. Time derivatives of integrals and functionals defined on varying volume and surface domains. Arch. Mech. 1986. 38:694-724.

[41] Simon J. Second variations for domain optimization problems. In F Kappel, K Kunisch, W Schappacher, editors, Control theory of distributed parameter systems and applications., volume 91 of International Series of Numerical Mathematics. Birkhäuser Verlag, Basel, 1989 $361-378$.

[42] Sokolowski J, Zolesio JP. Introduction to shape optimization. Shape sensitivity analysis, volume 16 of Springer series in Computational Mathematics. Springer-Verlag, 1992.

[43] Tanaka M, Sladek V, Sladek J. Regularization techniques applied to boundary element methods. Appl. Mech. Rev. 1994. 47:457-499.

[44] Tikhonov AN, Arsenin VY. Solutions to ill-posed problems. Winston-Wiley, New York, 1977.

[45] Zhang Q, Mukherjee S. Second-order design sensitivity analysis for linear elastic problems by the derivative boundary element method. Comp. Meth. in Appl. Mech. Engng. 1991. 86:321335.

\section{A Appendix}

\section{A.1 Elastodynamic fundamental solution}

The elastodynamic state created at $\boldsymbol{x} \in \mathbb{R}^{3}$ by a time-harmonic unit point force applied in an infinite elastic body at the fixed point $\tilde{\boldsymbol{x}}$ along the $\boldsymbol{e}_{k}$-direction is well-known; it is given below, both for completeness and in a form suitable for the regularization of the hypersingular kernel. The

fundamental displacement $U_{i}^{k}(\tilde{\boldsymbol{x}}, \boldsymbol{x})$, stress tensor $\Sigma_{i j}^{k}(\tilde{\boldsymbol{x}}, \boldsymbol{x})$ and traction vector $T_{i}^{k}(\tilde{\boldsymbol{x}}, \boldsymbol{x})$ are given 
by:

$$
\begin{aligned}
U_{i}^{k}(\tilde{\boldsymbol{x}}, \boldsymbol{x}) & =2(1-\nu)\left[F_{, a a}+k_{L}^{2} F\right] \delta_{i k}-F_{, i k} \\
\Sigma_{i j}^{k}(\tilde{\boldsymbol{x}}, \boldsymbol{x}) & =\mu\left[\frac{2 \nu}{1-2 \nu} \delta_{i j} U_{a, a}^{k}+U_{i, j}^{k}+U_{j, i}^{k}\right] \\
T_{i}^{k}(\tilde{\boldsymbol{x}}, \boldsymbol{x}) & =\Sigma_{i j}^{k} n_{j}
\end{aligned}
$$

in terms of the Somigliana potential [22] F:

$$
F(\tilde{\boldsymbol{x}}, \boldsymbol{x})=\frac{1}{4 \pi \mu k_{T}^{2}}\left(e^{i k_{L} r}-e^{i k_{T} r}\right) \frac{1}{r}
$$

$\left(k_{T}^{2}=\rho \omega^{2} / \mu\right.$ and $k_{L}^{2}=\kappa k_{T}^{2}$, with $\kappa=\frac{1-2 \nu}{2(1-\nu)}$ : transversal and longitudinal wave numbers, $r=$ $|\boldsymbol{x}-\tilde{\boldsymbol{x}}|$ : Euclidian distance between $\boldsymbol{x}, \tilde{\boldsymbol{x}})$. F satisfies the equation:

$$
F_{, a a b b}=\frac{k_{T}^{2}}{4 \pi \mu}\left(\kappa^{2} e^{i k_{L} r}-e^{i k_{T} r}\right) \frac{1}{r}
$$

In addition, the kernel $D_{i k}(\tilde{\boldsymbol{x}}, \boldsymbol{x})$ admits the following decomposition, which involves the surface curl defined by (12):

$$
\begin{aligned}
D_{i k}(\tilde{\boldsymbol{x}}, \boldsymbol{x}) & \equiv C_{i j a b} \Sigma_{k \ell, \bar{b}}^{a}(\tilde{\boldsymbol{x}}, \boldsymbol{x}) n_{j}(\tilde{\boldsymbol{x}}) n_{\ell}(\boldsymbol{x}) \\
& =R_{q}^{x} R_{s}^{y} B_{i k q s}(\tilde{\boldsymbol{x}}, \boldsymbol{x})+k_{T}^{2} A_{i k}(\tilde{\boldsymbol{x}}, \boldsymbol{x})
\end{aligned}
$$

where the kernel functions $B_{i k q s}(\tilde{\boldsymbol{x}}, \boldsymbol{x})$ and $A_{i k}(\tilde{\boldsymbol{x}}, \boldsymbol{x})$ are given by:

$$
\begin{aligned}
& B_{i k q s}(\tilde{\boldsymbol{x}}, \boldsymbol{x})=-e_{i e p} e_{k g r} \mu^{2}\left[4 \nu \delta_{p q} \delta_{r s}+2(1-\nu)\left(\delta_{p r} \delta_{q s}+\delta_{p s} \delta_{q r}\right)\right] F_{, e g} \\
& A_{i k}(\tilde{\boldsymbol{x}}, \boldsymbol{x})=\mu^{2} A_{i j k \ell}(\tilde{\boldsymbol{x}}, \boldsymbol{x}) n_{j}(\tilde{\boldsymbol{x}}) n_{\ell}(\boldsymbol{x}) \\
& A_{i j k \ell}(\tilde{\boldsymbol{x}}, \boldsymbol{x})=k_{T}^{2}\left[\frac{4 \nu^{2}}{1-2 \nu} \delta_{i j} \delta_{k \ell} F_{, a a}+4 \nu\left(\delta_{i j} F_{, k \ell}+\delta_{k \ell}\right) F_{, i j}\right. \\
&\left.+(1-2 \nu)\left(\delta_{i k} F_{, j \ell}+\delta_{j \ell} F_{, i k}+\delta_{j k} F_{, i \ell}+\delta_{i \ell} F_{, j k}\right)\right] \\
&+\left[2(1-\nu)\left(\delta_{i k} \delta_{j \ell}+\delta_{j k} \delta_{i \ell}\right)+\frac{2 \nu}{\kappa} \delta_{i j} \delta_{k \ell}\right] F_{, a a b b}
\end{aligned}
$$

and are both weakly singular in view of Eqs. (85) and (86).

\section{A.2 Proof of result (20)}

Applying the material derivative formulas (14), (15) to identity (16) gives:

$$
\begin{aligned}
& \int_{S_{\varepsilon}+s_{\varepsilon}}\left\{T_{a}^{k}(\tilde{\boldsymbol{x}}, \boldsymbol{x}) \stackrel{\star}{u}_{a}(\boldsymbol{x})-U_{a}^{k}(\tilde{\boldsymbol{x}}, \boldsymbol{x}) \stackrel{\star}{p}_{a}(\boldsymbol{x})\right\} d S_{x} \\
& \quad+\int_{S_{\varepsilon}+s_{\varepsilon}} u_{a}(\boldsymbol{x})\left\{\Sigma_{a b, c}^{k}(\tilde{\boldsymbol{x}}, \boldsymbol{x})\left[\theta_{c}(\boldsymbol{x})-\theta_{c}(\tilde{\boldsymbol{x}})\right] n_{b}(\boldsymbol{x})+e_{b c i} \Sigma_{a b}^{k}(\tilde{\boldsymbol{x}}, \boldsymbol{x}) R_{i} \theta_{c}(\boldsymbol{x})\right\} d S_{x} \\
& \quad-\int_{S_{\varepsilon}+s_{\varepsilon}}\left\{U_{a, c}^{k}(\tilde{\boldsymbol{x}}, \boldsymbol{x})\left[\theta_{c}(\boldsymbol{x})-\theta_{c}(\tilde{\boldsymbol{x}})\right]+U_{a}^{k}(\tilde{\boldsymbol{x}}, \boldsymbol{x}) D_{c} \theta_{c}(\boldsymbol{x})\right\} p_{a}(\boldsymbol{x}) d S_{x}=0
\end{aligned}
$$


where formulas $(7),(8)$ have been taken into account, together with the relations:

$$
\begin{gathered}
{\stackrel{\star}{\Sigma_{a b}}}^{k}(\tilde{\boldsymbol{x}}, \boldsymbol{x})=\left[\theta_{c}(\boldsymbol{x})-\theta_{c}(\tilde{\boldsymbol{x}})\right] \Sigma_{a b, c}^{k}(\tilde{\boldsymbol{x}}, \boldsymbol{x}) \\
\stackrel{\star}{U_{a}^{k}}(\tilde{\boldsymbol{x}}, \boldsymbol{x})=\left[\theta_{c}(\boldsymbol{x})-\theta_{c}(\tilde{\boldsymbol{x}})\right] U_{a, c}^{k}(\tilde{\boldsymbol{x}}, \boldsymbol{x})
\end{gathered}
$$

Next, expression (91) can be conveniently rearranged using identity (100):

$$
\begin{aligned}
& \int_{S_{\varepsilon}+s_{\varepsilon}} u_{a}(\boldsymbol{x})\left\{\Sigma_{a b, c}^{k}(\tilde{\boldsymbol{x}}, \boldsymbol{x})\left[\theta_{c}(\boldsymbol{x})-\theta_{c}(\tilde{\boldsymbol{x}})\right] n_{b}(\boldsymbol{x})+\Sigma_{a b}^{k}(\tilde{\boldsymbol{x}}, \boldsymbol{x}) e_{b c i} R_{i} \theta_{c}(\boldsymbol{x})\right\} d S_{x} \\
& =\int_{S_{\varepsilon}+s_{\varepsilon}}\left\{\Sigma_{a b}^{k}(\tilde{\boldsymbol{x}}, \boldsymbol{x}) e_{c b i} R_{i} u_{a}(\boldsymbol{x})-\rho \omega^{2} U_{a}^{k}(\tilde{\boldsymbol{x}}, \boldsymbol{x}) u_{a}(\boldsymbol{x}) n_{c}(\boldsymbol{x})\right\}\left[\theta_{c}(\boldsymbol{x})-\theta_{c}(\tilde{\boldsymbol{x}})\right] d S_{x}
\end{aligned}
$$

where the differential operator $R_{i}$ is understood as acting on the variable $\boldsymbol{x}$. Substitution of eq. (94) into (91) finally yields Eq. (20).

\section{A.3 Proof of the hypersingular BIE (23)}

First, upon appropriately grouping terms, one finds (using the property $(U, \Sigma)_{, \bar{b}}=-(U, \Sigma)_{, b}$ verified by the infinite-space fundamental solutions) that:

$$
\begin{aligned}
& u_{k, c}(\tilde{\boldsymbol{x}})\left(y_{c}-x_{c}\right) \Sigma_{k \ell, \bar{b}}^{a}(\tilde{\boldsymbol{x}}, \boldsymbol{x}) n_{\ell}(\boldsymbol{x})-\sigma_{k \ell}(\tilde{\boldsymbol{x}}) n_{\ell}(\boldsymbol{x}) U_{k, \bar{b}}^{a}(\tilde{\boldsymbol{x}}, \boldsymbol{x}) \\
& =-u_{k, c}(\tilde{\boldsymbol{x}}) e_{\ell b d} R_{d}\left(\left(y_{c}-x_{c}\right) \Sigma_{k \ell}^{a}(\tilde{\boldsymbol{x}}, \boldsymbol{x})\right)+\sigma_{k \ell}(\tilde{\boldsymbol{x}}) e_{\ell b d} R_{d} U_{k}^{a}(\tilde{\boldsymbol{x}}, \boldsymbol{x}) \\
& \quad+u_{k, \bar{b}}(\tilde{\boldsymbol{x}}) T_{k}^{a}(\tilde{\boldsymbol{x}}, \boldsymbol{x})+\rho \omega^{2} u_{k, c}(\tilde{\boldsymbol{x}}) n_{b}(\boldsymbol{x})\left(y_{c}-x_{c}\right) U_{k}^{a}(\tilde{\boldsymbol{x}}, \boldsymbol{x}) \\
& \quad-u_{a, b}(\tilde{\boldsymbol{x}}) n_{b}(\boldsymbol{x}) \Sigma_{k \ell}^{a}(\tilde{\boldsymbol{x}}, \boldsymbol{x})+\sigma_{k \ell}(\tilde{\boldsymbol{x}}) n_{b}(\boldsymbol{x}) U_{k, \ell}^{a}(\tilde{\boldsymbol{x}}, \boldsymbol{x})
\end{aligned}
$$

Moreover, the symmetry of the elastic constitutive relation implies that the last two terms in the right-hand side cancel each other.

One then has:

$$
\begin{aligned}
& \int_{s_{\varepsilon}}\left\{u_{k}(\boldsymbol{x}) \Sigma_{k \ell, \bar{b}}^{a}(\tilde{\boldsymbol{x}}, \boldsymbol{x}) n_{\ell}(\boldsymbol{x})-p_{k}(\boldsymbol{x}) U_{k, \bar{b}}^{a}(\tilde{\boldsymbol{x}}, \boldsymbol{x})\right\} d S_{x} \\
& =\int_{s_{\varepsilon}}\left[u_{k}(\boldsymbol{x})-u_{k}(\tilde{\boldsymbol{x}})-\left(y_{c}-x_{c}\right) u_{k, c}(\tilde{\boldsymbol{x}})\right] \Sigma_{k \ell, \bar{b}}^{a}(\tilde{\boldsymbol{x}}, \boldsymbol{x}) n_{\ell}(\boldsymbol{x}) d S_{x}+u_{k}(\tilde{\boldsymbol{x}}) \int_{s_{\varepsilon}} \Sigma_{k \ell, \bar{b}}^{a}(\tilde{\boldsymbol{x}}, \boldsymbol{x}) n_{\ell}(\boldsymbol{x}) d S_{x} \\
& \quad-\int_{s_{\varepsilon}}\left[\sigma_{k \ell}(\boldsymbol{x})-\sigma_{k \ell}(\tilde{\boldsymbol{x}})\right] n_{\ell}(\boldsymbol{x}) U_{k, \bar{b}}^{a}(\tilde{\boldsymbol{x}}, \boldsymbol{x}) d S_{x} \\
& \quad+\sigma_{k \ell}(\tilde{\boldsymbol{x}}) e_{\ell b d} \int_{c_{\varepsilon}} \tau_{d} U_{k}^{a}(\tilde{\boldsymbol{x}}, \boldsymbol{x}) d s_{x}+e_{b \ell d} u_{k, c}(\tilde{\boldsymbol{x}}) \int_{c_{\varepsilon}} \tau_{d}\left(y_{c}-x_{c}\right) \Sigma_{k \ell}^{a}(\tilde{\boldsymbol{x}}, \boldsymbol{x}) d s_{x} \\
& \quad+\rho \omega^{2} u_{k, c}(\tilde{\boldsymbol{x}}) \int_{s_{\varepsilon}} U_{k}^{a}(\tilde{\boldsymbol{x}}, \boldsymbol{x}) n_{b}(\boldsymbol{x})\left(y_{c}-x_{c}\right) d S_{x}+u_{k, b}(\tilde{\boldsymbol{x}}) \int_{s_{\varepsilon}} T_{k}^{a}(\tilde{\boldsymbol{x}}, \boldsymbol{x}) d S_{x}
\end{aligned}
$$

To obtain this identity, the first two terms of the right-hand side of (95) have been integrated by parts using formula (13). 
The first two integrals in the right-hand side of Eq. (96) vanish in the limit due to the assumed smoothness of $\boldsymbol{u}$ and $\boldsymbol{\sigma}$ at $\tilde{\boldsymbol{x}}$, and the next-to-last does because $U_{k}^{a}=O\left(r^{-1}\right)$. Moreover, if a spherical exclusion neighbourhood $v_{\varepsilon}$ is used, the two contour integrals are found to vanish in the limit. Finally, the last integral of Eq. (96) is given by Eq. (19). One therefore has:

$$
\begin{aligned}
\int_{s_{\varepsilon}}\left\{u_{k}(\boldsymbol{x}) \Sigma_{k \ell, \bar{b}}^{a}(\tilde{\boldsymbol{x}}, \boldsymbol{x}) n_{\ell}(\boldsymbol{x})-p_{k}(\boldsymbol{x}) U_{k, \bar{b}}^{a}(\tilde{\boldsymbol{x}}, \boldsymbol{x})\right\} d S_{x} & \\
& =\frac{1}{2} u_{a, b}(\tilde{\boldsymbol{x}})+u_{k}(\tilde{\boldsymbol{x}}) \int_{s_{\varepsilon}} \Sigma_{k \ell, \bar{b}}^{a}(\tilde{\boldsymbol{x}}, \boldsymbol{x}) n_{\ell}(\boldsymbol{x}) d S_{x}+O(\varepsilon)
\end{aligned}
$$

The hypersingular BIE (23) then readily follows from the previous argument and the definition $(25)$.

\section{A.4 Proof of result (27)}

After having applied identity (101) to the second integral of (26) and upon expanding and regrouping terms in the resulting equality in anticipation of the final limiting process, one obtains:

$$
\begin{aligned}
& \int_{S_{\varepsilon}}\left\{\Sigma_{k \ell, \bar{b}}^{a}(\tilde{\boldsymbol{x}}, \boldsymbol{x}) n_{\ell}(\boldsymbol{x}) \stackrel{\star}{u_{k}}(\boldsymbol{x})-U_{k, \bar{b}}^{a}(\tilde{\boldsymbol{x}}, \boldsymbol{x}) \stackrel{\star}{p_{k}}(\boldsymbol{x})\right\} d S_{x} \\
& +\stackrel{\star}{u}_{k}(\tilde{\boldsymbol{x}}) \int_{s_{\varepsilon}} \Sigma_{k \ell, \bar{b}}^{a}(\tilde{\boldsymbol{x}}, \boldsymbol{x}) n_{\ell}(\boldsymbol{x}) d S_{x} \\
& +\int_{S_{\varepsilon}} e_{c \ell d} R_{d} u_{k}(\boldsymbol{x}) \Sigma_{k \ell, \bar{b}}^{a}(\tilde{\boldsymbol{x}}, \boldsymbol{x})\left[\theta_{c}(\boldsymbol{x})-\theta_{c}(\tilde{\boldsymbol{x}})\right] d S_{x} \\
& -\rho \omega^{2} \int_{S_{\varepsilon}} u_{k}(\boldsymbol{x}) U_{k, \bar{b}}^{a}(\tilde{\boldsymbol{x}}, \boldsymbol{x})\left[\theta_{c}(\boldsymbol{x})-\theta_{c}(\tilde{\boldsymbol{x}})\right] n_{c}(\boldsymbol{x}) d S_{x} \\
& -\int_{S_{\varepsilon}}\left\{U_{k, \bar{b} c}^{a}(\tilde{\boldsymbol{x}}, \boldsymbol{x})\left[\theta_{c}(\boldsymbol{x})-\theta_{c}(\tilde{\boldsymbol{x}})\right]+U_{k, \bar{b}}^{a}(\tilde{\boldsymbol{x}}, \boldsymbol{x}) D_{c} \theta_{c}\right\} p_{k}(\boldsymbol{x}) d S_{x} \\
& +\int_{s_{\varepsilon}} \Sigma_{k \ell, \bar{b}}^{a}(\tilde{\boldsymbol{x}}, \boldsymbol{x}) n_{\ell}(\boldsymbol{x})\left[\stackrel{\star}{u}_{k}(\boldsymbol{x})-\stackrel{\star}{u}_{k}(\tilde{\boldsymbol{x}})-\left(y_{d}-x_{d}\right) \stackrel{\star}{u_{k, d}}(\tilde{\boldsymbol{x}})\right] d S_{x} \quad \quad(=O(\varepsilon)) \\
& -\int_{s_{\varepsilon}} U_{k, \bar{b}}^{a}(\tilde{\boldsymbol{x}}, \boldsymbol{x})\left[\stackrel{\star}{\sigma}_{k \ell}(\boldsymbol{x})-\stackrel{\star}{\sigma}_{k \ell}(\tilde{\boldsymbol{x}})\right] n_{\ell}(\boldsymbol{x}) d S_{x} \quad \quad(=O(\varepsilon)) \\
& +\int_{s_{\varepsilon}}\left\{\Sigma_{k \ell, \bar{b}}^{a}(\tilde{\boldsymbol{x}}, \boldsymbol{x})\left(y_{d}-x_{d}\right) \stackrel{\star}{u}_{k, d}(\tilde{\boldsymbol{x}})-U_{k, \bar{b}}^{a}(\tilde{\boldsymbol{x}}, \boldsymbol{x}) \stackrel{\star}{\sigma}_{k \ell}(\tilde{\boldsymbol{x}})\right\} n_{\ell}(\boldsymbol{x}) d S_{x} \\
& +\int_{s_{\varepsilon}} e_{c \ell d} R_{d} u_{k}(\boldsymbol{x}) \Sigma_{k \ell, \bar{b}}^{a}(\tilde{\boldsymbol{x}}, \boldsymbol{x})\left[\theta_{c}(\boldsymbol{x})-\theta_{c}(\tilde{\boldsymbol{x}})\right] d S_{x} \\
& -\rho \omega^{2} \int_{s_{\varepsilon}} u_{k}(\boldsymbol{x}) U_{k, \bar{b}}^{a}(\tilde{\boldsymbol{x}}, \boldsymbol{x}) n_{c}(\boldsymbol{x})\left[\theta_{c}(\boldsymbol{x})-\theta_{c}(\tilde{\boldsymbol{x}})\right] d S_{x} \quad \quad(=O(\varepsilon)) \\
& -\int_{s_{\varepsilon}}\left\{U_{k, \bar{b} c}^{a}(\tilde{\boldsymbol{x}}, \boldsymbol{x})\left[\theta_{c}(\boldsymbol{x})-\theta_{c}(\tilde{\boldsymbol{x}})\right] n_{\ell}(\boldsymbol{x})+U_{k, \bar{b}}^{a}(\tilde{\boldsymbol{x}}, \boldsymbol{x}) D_{\ell c} \theta_{c}\right\} \sigma_{k \ell}(\boldsymbol{x}) d S_{x}=0
\end{aligned}
$$

where terms known to vanish in the limit $\varepsilon \rightarrow 0$ have been indicated.

In Eq. (98), the first five lines will lead to FP and CPV integrals over $S$ and will not be modified further, whereas the sixth and seventh vanish in the limit $\varepsilon \rightarrow 0$. Let $I_{k \ell}(\tilde{\boldsymbol{x}}, \varepsilon)$ denote the sum of 
integrals over $s_{\varepsilon}$ appearing in (98). The limiting value of $I_{k \ell}(\tilde{\boldsymbol{x}}, \varepsilon)$ remains to be investigated. From Eq. (98), one has:

$$
I_{k \ell}(\tilde{\boldsymbol{x}}, \varepsilon)=\int_{s_{\varepsilon}} E_{k \ell}(\tilde{\boldsymbol{x}}, \boldsymbol{x}) d s_{x}+O(\varepsilon)
$$

with

$$
\begin{aligned}
& \left\{\Sigma_{k \ell, \bar{b}}^{a}(\tilde{\boldsymbol{x}}, \boldsymbol{x})\left(y_{d}-x_{d}\right){\stackrel{\star}{u_{k, d}}}^{(\tilde{\boldsymbol{x}})}-U_{k, \bar{b}}^{a}(\tilde{\boldsymbol{x}}, \boldsymbol{x}) \stackrel{\star}{\sigma}_{k \ell}(\tilde{\boldsymbol{x}})\right\} n_{\ell}(\boldsymbol{x}) \\
& \quad+e_{c \ell d} R_{d} u_{k}(\boldsymbol{x}) \Sigma_{k \ell, \bar{b}}^{a}(\tilde{\boldsymbol{x}}, \boldsymbol{x})\left[\theta_{c}(\boldsymbol{x})-\theta_{c}(\tilde{\boldsymbol{x}})\right] \\
& \quad-\left\{U_{k, \bar{b} c}^{a}(\tilde{\boldsymbol{x}}, \boldsymbol{x})\left[\theta_{c}(\boldsymbol{x})-\theta_{c}(\tilde{\boldsymbol{x}})\right] n_{\ell}(\boldsymbol{x})+U_{k, \bar{b}}^{a}(\tilde{\boldsymbol{x}}, \boldsymbol{x}) e_{\ell c d} R_{d} \theta_{c}\right\} \sigma_{k \ell}(\boldsymbol{x})
\end{aligned}
$$

First, using property (6) of the material derivative and performing some subtractions and additions aimed at taking advantage of the assumed $C^{0, \alpha}$ smoothness of $\boldsymbol{\nabla} \boldsymbol{u}$ and $\boldsymbol{\sigma}$ at $\boldsymbol{x}=\tilde{\boldsymbol{x}}$, one finds:

$$
\begin{aligned}
& E_{k \ell}(\tilde{\boldsymbol{x}}, \boldsymbol{x})=\left\{\Sigma_{k \ell, \bar{b}}^{a}(\tilde{\boldsymbol{x}}, \boldsymbol{x})\left(y_{d}-x_{d}\right)\left(u_{k, d}\right)^{\star}(\tilde{\boldsymbol{x}})-U_{k, \bar{b}}^{a}(\tilde{\boldsymbol{x}}, \boldsymbol{x}) \stackrel{\star}{\sigma}_{k \ell}(\tilde{\boldsymbol{x}})\right\} n_{\ell}(\boldsymbol{x}) \\
& +\left[n_{c}(\boldsymbol{x})\left[u_{k, \ell}(\boldsymbol{x})-u_{k, \ell}(\tilde{\boldsymbol{x}})\right]-n_{\ell}(\boldsymbol{x})\left[u_{k, c}(\boldsymbol{x})-u_{k, c}(\tilde{\boldsymbol{x}})\right]\right] \Sigma_{k \ell, \bar{b}}^{a}(\tilde{\boldsymbol{x}}, \boldsymbol{x})\left[\theta_{c}(\boldsymbol{x})-\theta_{c}(\tilde{\boldsymbol{x}})\right] \\
& \left(=O\left(\varepsilon^{\alpha-2}\right)\right) \\
& +\left[n_{c}(\boldsymbol{x}) u_{k, \ell}(\tilde{\boldsymbol{x}})-n_{\ell}(\boldsymbol{x}) u_{k, c}(\tilde{\boldsymbol{x}})\right] \Sigma_{k \ell, \bar{b}}^{a}(\tilde{\boldsymbol{x}}, \boldsymbol{x})\left[\theta_{c}(\boldsymbol{x})-\theta_{c}(\tilde{\boldsymbol{x}})\right] \quad \quad\left(=O\left(\varepsilon^{\alpha-2}\right)\right) \\
& +u_{k, c}(\tilde{\boldsymbol{x}}) \theta_{c, d}(\tilde{\boldsymbol{x}}) \Sigma_{k \ell, \bar{b}}^{a}(\tilde{\boldsymbol{x}}, \boldsymbol{x})\left(y_{d}-x_{d}\right) n_{\ell}(\boldsymbol{x}) \\
& -\left\{U_{k, \bar{b} c}^{a}(\tilde{\boldsymbol{x}}, \boldsymbol{x})\left[\theta_{c}(\boldsymbol{x})-\theta_{c}(\tilde{\boldsymbol{x}})\right] n_{\ell}(\boldsymbol{x})+U_{k, \bar{b}}^{a}(\tilde{\boldsymbol{x}}, \boldsymbol{x}) e_{\ell c d} R_{d} \theta_{c}\right\}\left[\sigma_{k \ell}(\boldsymbol{x})-\sigma_{k \ell}(\tilde{\boldsymbol{x}})\right] \\
& \left(=O\left(\varepsilon^{\alpha-2}\right)\right) \\
& -\left\{U_{k, \bar{b} c}^{a}(\tilde{\boldsymbol{x}}, \boldsymbol{x})\left[\theta_{c}(\boldsymbol{x})-\theta_{c}(\tilde{\boldsymbol{x}})\right] n_{\ell}(\boldsymbol{x})+U_{k, \bar{b}}^{a}(\tilde{\boldsymbol{x}}, \boldsymbol{x}) e_{\ell c d} R_{d} \theta_{c}\right\} \sigma_{k \ell}(\tilde{\boldsymbol{x}}) \\
& =\left\{\Sigma_{k \ell, \bar{b}}^{a}(\tilde{\boldsymbol{x}}, \boldsymbol{x})\left(y_{d}-x_{d}\right)\left(u_{k, d}\right)^{\star}(\tilde{\boldsymbol{x}})-U_{k, \bar{b}}^{a}(\tilde{\boldsymbol{x}}, \boldsymbol{x}) \stackrel{\star}{\sigma}_{k \ell}(\tilde{\boldsymbol{x}})\right\} n_{\ell}(\boldsymbol{x}) \\
& +n_{c}(\boldsymbol{x}) \sigma_{k \ell}(\tilde{\boldsymbol{x}}) U_{k, \ell \bar{b}}^{a}(\tilde{\boldsymbol{x}}, \boldsymbol{x})\left[\theta_{c}(\boldsymbol{x})-\theta_{c}(\tilde{\boldsymbol{x}})\right]-n_{\ell}(\boldsymbol{x}) u_{k, c}(\tilde{\boldsymbol{x}}) \Sigma_{k \ell, \bar{b}}^{a}(\tilde{\boldsymbol{x}}, \boldsymbol{x})\left[\theta_{c}(\boldsymbol{x})-\theta_{c}(\tilde{\boldsymbol{x}})\right] \\
& +u_{k, c}(\tilde{\boldsymbol{x}}) \theta_{c, d}(\tilde{\boldsymbol{x}}) \Sigma_{k \ell, \bar{b}}^{a}(\tilde{\boldsymbol{x}}, \boldsymbol{x})\left(y_{d}-x_{d}\right) n_{\ell}(\boldsymbol{x}) \\
& -\left\{U_{k, \bar{b} c}^{a}(\tilde{\boldsymbol{x}}, \boldsymbol{x})\left[\theta_{c}(\boldsymbol{x})-\theta_{c}(\tilde{\boldsymbol{x}})\right] n_{\ell}(\boldsymbol{x})+U_{k, \bar{b}}^{a}(\tilde{\boldsymbol{x}}, \boldsymbol{x}) e_{\ell c d} R_{d} \theta_{c}\right\} \sigma_{k \ell}(\tilde{\boldsymbol{x}})+O\left(\varepsilon^{\alpha-2}\right) \\
& =\left\{\Sigma_{k \ell, \bar{b}}^{a}(\tilde{\boldsymbol{x}}, \boldsymbol{x})\left(y_{d}-x_{d}\right)\left(u_{k, d}\right)^{\star}(\tilde{\boldsymbol{x}})-U_{k, \bar{b}}^{a}(\tilde{\boldsymbol{x}}, \boldsymbol{x}) \stackrel{\star}{\sigma}_{k \ell}(\tilde{\boldsymbol{x}})\right\} n_{\ell}(\boldsymbol{x}) \\
& -n_{\ell}(\boldsymbol{x}) u_{k, c}(\tilde{\boldsymbol{x}}) \Sigma_{k \ell, \bar{b}}^{a}(\tilde{\boldsymbol{x}}, \boldsymbol{x})\left[\theta_{c}(\boldsymbol{x})-\theta_{c}(\tilde{\boldsymbol{x}})-\theta_{c, d}(\tilde{\boldsymbol{x}})\right] \\
& -\sigma_{k \ell}(\tilde{\boldsymbol{x}}) e_{\ell c d} R_{d}\left\{U_{k, \bar{b}}^{a}(\tilde{\boldsymbol{x}}, \boldsymbol{x})\left[\theta_{c}(\boldsymbol{x})-\theta_{c}(\tilde{\boldsymbol{x}})\right]\right\}+O\left(\varepsilon^{\alpha-2}\right)
\end{aligned}
$$

where the assumption $\boldsymbol{\theta} \in C^{1, \alpha}$ at $\boldsymbol{x}=\tilde{\boldsymbol{x}}$ has been used, as well as the condition $\boldsymbol{u} \in C^{1, \alpha}$. Next, using again identity $(95)$ with $u_{k, d}(\tilde{\boldsymbol{x}}), \sigma_{k \ell}(\tilde{\boldsymbol{x}})$ replaced by $\left(u_{k, d}\right)^{\star}(\tilde{\boldsymbol{x}}), \stackrel{\star}{\sigma}_{k \ell}(\tilde{\boldsymbol{x}})$ (and in particular noting that the symmetry of the elastic constitutive relation implies the equality $\left(u_{k, \ell}\right)^{\star} \Sigma_{k \ell}^{a}=\stackrel{\stackrel{\sigma}{\sigma}}{k \ell}^{\star}$ 
$\left.U_{k, \ell}^{a}\right)$ yields:

$$
\begin{aligned}
E_{k \ell}(\tilde{\boldsymbol{x}}, \boldsymbol{x})= & -\left(u_{k, c}\right)^{\star}(\tilde{\boldsymbol{x}}) e_{\ell b d} R_{d}\left(\left(y_{c}-x_{c}\right) \Sigma_{k \ell}^{a}(\tilde{\boldsymbol{x}}, \boldsymbol{x})\right)+\stackrel{\star}{\sigma}_{k \ell} e_{\ell b d} R_{d} U_{k}^{a}(\tilde{\boldsymbol{x}}, \boldsymbol{x}) \\
& +u_{k, b}^{\star}(\tilde{\boldsymbol{x}}) T_{k}^{a}(\tilde{\boldsymbol{x}}, \boldsymbol{x})-\sigma_{k \ell}(\tilde{\boldsymbol{x}}) e_{\ell c d} R_{d}\left\{U_{k, \bar{b}}^{a}(\tilde{\boldsymbol{x}}, \boldsymbol{x})\left[\theta_{c}(\boldsymbol{x})-\theta_{c}(\tilde{\boldsymbol{x}})\right]\right\}+O\left(\varepsilon^{\alpha-2}\right)
\end{aligned}
$$

Integrating this equality over $s_{\varepsilon}$, using the Stokes identity (13) and noting that since $d S_{x} \sim \varepsilon^{2} \mathrm{~d} A$ ( $A$ : solid angle with origin $\tilde{\boldsymbol{x}}$ ), the $O\left(\varepsilon^{\alpha-2}\right)$ remainder gives rise to a $O(\varepsilon)$, one finds:

$$
\begin{aligned}
I_{k \ell}(\tilde{\boldsymbol{x}}, \varepsilon)= & -\left(u_{k, c}\right)^{\star}(\tilde{\boldsymbol{x}}) e_{\ell b d} \int_{c_{\varepsilon}} \tau_{d}(\boldsymbol{x})\left(y_{c}-x_{c}\right) \Sigma_{k \ell}^{a}(\tilde{\boldsymbol{x}}, \boldsymbol{x}) d s_{x}+\stackrel{\star}{\sigma}_{k \ell}(\tilde{\boldsymbol{x}}) e_{\ell b d} \int_{c_{\varepsilon}} \tau_{d}(\boldsymbol{x}) U_{k}^{a}(\tilde{\boldsymbol{x}}, \boldsymbol{x}) d s_{x} \\
& -\sigma_{k \ell}(\tilde{\boldsymbol{x}}) e_{\ell c d} \int_{c_{\varepsilon}} \tau_{d}(\boldsymbol{x}) U_{k, \bar{b}}^{a}(\tilde{\boldsymbol{x}}, \boldsymbol{x})\left[\theta_{c}(\boldsymbol{x})-\theta_{c}(\tilde{\boldsymbol{x}})\right] d s_{x} \\
& +u_{k, b}^{\star}(\tilde{\boldsymbol{x}}) \int_{s_{\varepsilon}} T_{k}^{a}(\tilde{\boldsymbol{x}}, \boldsymbol{x}) d S_{x}+O\left(\varepsilon^{\alpha-2}\right)
\end{aligned}
$$

Equations (98) and (99) finally yield the desired result, Eq. (27).

\section{A.5 Some auxiliary identities}

Identities involving integration by parts. Let $S$ denote a piecewise smooth closed surface and $\tilde{\boldsymbol{x}}$ any point not located on $S$. Then, the equality:

$$
\begin{aligned}
u_{k}(\boldsymbol{x})\left\{\Sigma_{k \ell, c}^{a}(\tilde{\boldsymbol{x}}, \boldsymbol{x})\left[\theta_{c}(\boldsymbol{x})-\theta_{c}(\tilde{\boldsymbol{x}})\right] n_{\ell}(\boldsymbol{x})+\Sigma_{k \ell}^{a}(\tilde{\boldsymbol{x}}, \boldsymbol{x}) e_{\ell c i} R_{i} \theta_{c}(\boldsymbol{x})\right\} \\
\quad=e_{\ell c d} u_{k}(\boldsymbol{x}) R_{d}\left\{\Sigma_{k \ell}^{a}(\tilde{\boldsymbol{x}}, \boldsymbol{x})\left[\theta_{c}(\boldsymbol{x})-\theta_{c}(\tilde{\boldsymbol{x}})\right]\right\}-\rho \omega^{2} u_{k}(\boldsymbol{x}) U_{k}^{a}(\tilde{\boldsymbol{x}}, \boldsymbol{x})\left[\theta_{c}(\boldsymbol{x})-\theta_{c}(\tilde{\boldsymbol{x}})\right] n_{c}(\boldsymbol{x})
\end{aligned}
$$

holds true by virtue of the governing equation $\Sigma_{k \ell, \ell}^{a}+\rho \omega^{2} U_{k}^{a}=0$ verified by the fundamental solution for any $\boldsymbol{x} \neq \tilde{\boldsymbol{x}}$. Upon integration over $S$ and application of the Stokes formula (13) to the first term, one obtains the identity:

$$
\begin{aligned}
& \int_{S} u_{k}(\boldsymbol{x})\left\{\Sigma_{k \ell, c}^{a}(\tilde{\boldsymbol{x}}, \boldsymbol{x})\right. {\left.\left[\theta_{c}(\boldsymbol{x})-\theta_{c}(\tilde{\boldsymbol{x}})\right] n_{\ell}(\boldsymbol{x})+\Sigma_{k \ell}^{a}(\tilde{\boldsymbol{x}}, \boldsymbol{x}) e_{\ell c i} R_{i} \theta_{c}(\boldsymbol{x})\right\} d S_{x} } \\
&=\int_{S}\left\{e_{\ell d c} R_{d} u_{k}(\boldsymbol{x}) \Sigma_{k \ell}^{a}(\tilde{\boldsymbol{x}}, \boldsymbol{x})-\rho \omega^{2} U_{k}^{a}(\tilde{\boldsymbol{x}}, \boldsymbol{x}) n_{c}(\boldsymbol{x})\right\}\left[\theta_{c}(\boldsymbol{x})-\theta_{c}(\tilde{\boldsymbol{x}})\right] d S_{x}
\end{aligned}
$$

Repeating the same argument with $\Sigma_{k \ell}^{a}$ replaced by $\Sigma_{k \ell, \bar{b}}^{a}$ (and in particular using the equality $\left.\Sigma_{k \ell, \bar{b} \ell}^{a}+\rho \omega^{2} U_{k, \bar{b}}^{a}=0\right)$, one obtains the identity:

$$
\begin{aligned}
\int_{S} u_{k}(\boldsymbol{x})\left\{\Sigma_{k \ell, \bar{b} c}^{a}(\tilde{\boldsymbol{x}}, \boldsymbol{x})\left[\theta_{c}(\boldsymbol{x})-\theta_{c}(\tilde{\boldsymbol{x}})\right] n_{\ell}(\boldsymbol{x})+\Sigma_{k \ell, \bar{b}}^{a}(\tilde{\boldsymbol{x}}, \boldsymbol{x}) e_{\ell c i} R_{i} \theta_{c}(\boldsymbol{x})\right\} d S_{x} \\
=\int_{S}\left\{e_{\ell d c} R_{d} u_{k}(\boldsymbol{x}) \Sigma_{k \ell, \bar{b}}^{a}(\tilde{\boldsymbol{x}}, \boldsymbol{x})-\rho \omega^{2} U_{k, \bar{b}}^{a}(\tilde{\boldsymbol{x}}, \boldsymbol{x}) n_{c}(\boldsymbol{x})\right\}\left[\theta_{c}(\boldsymbol{x})-\theta_{c}(\tilde{\boldsymbol{x}})\right] d S_{x}
\end{aligned}
$$


Proof of equation (62). Using Eqs. (6) and (11), one has:

$$
\begin{aligned}
\left(R_{i} u \mathrm{~d} S\right)^{\star}=e_{i j k}\left(e_{j a b} R_{b} \theta_{a} u_{, k}-n_{j} u_{a} \theta_{a, k}\right) \mathrm{d} S=\left(R_{i} \theta_{k}-R_{k} \theta_{i}\right) u_{, k}-R_{i} \theta_{a} u_{, a} & \\
& =-R_{a} \theta_{i} u_{, a}=R_{a} u D_{a} \theta_{i}
\end{aligned}
$$

i.e. Eq. (62). The last equality results from invoking again the definition (12) of the surface curl and its consequence $n_{a} R_{a} u=0$. 